\title{
Anmerkungen zu einigen Dresdner Notisten im Lichte der Überlieferung von Opern Johann Adolf Hasses*
}

Die Wirkungszeit Johann Adolf Hasses am Dresdner Hof, dessen Musikleben er maßgeblich mitbestimmte, spannt sich über mehr als 30 Jahre. Erste Verhandlungen über ein Engagement nach Dresden hatten schon im Frühjahr 1730 kurz vor dem Abschluss gestanden, führten dann aber doch nicht zu einer sofortigen Verpflichtung Hasses an die Elbe, da dieser sich offenbar bei seiner Hochzeit mit der venezianischen Primadonna Faustina Bordoni hatte verpflichten müssen, einstweilen in Italien zu bleiben, um Faustinas dortige Impegni nicht zu gefährden. ${ }^{1}$ Erst im Spätsommer 1731 kamen die Hasses zu einem Gastspiel nach Dresden, das am 13. September des Jahres von der glanzvollen Erstaufführung der Oper Cleofide gekrönt wurde. Ende 1733 schließlich trat das Ehepaar dann offiziell in die Dienste von König August III. (als Kurfürst Friedrich August II.), als dessen Kapellmeister Hasse bis zum Regierungswechsel 1763 amtierte, während sich Faustina 1751 von der Opernbühne zurückzog. Allerdings erhielt Hasse während der Zeiten, in denen der Hof in Warschau weilte, regelmäßig Urlaub, den er meist zu Reisen nach Italien nutzte; auch während des Siebenjährigen Krieges hielt er sich - abgesehen von einem Besuch in Warschau im Herbst und Winter 1762 - überwiegend in Italien bzw. ab 1760 in Wien auf. Hasses Schaffensschwerpunkt in Dresden war die repräsentative Vokalmusik, das heißt die Opera seria, das Oratorium und die groß besetzte Kirchenmusik. Hingegen dürfte Instrumentalmusik zumindest in Hasses Dresdner Cuvre nur eine untergeordnete Rolle gespielt haben, worauf nicht zuletzt die Tatsache verweist, dass in den Beständen der SLUB Dresden nur wenige Instrumentalwerke unter Hasses Namen über-

* Eine etwas überarbeitete Fassung des vorliegenden Aufsatzes erschien im Jahrbuch des Staatlichen Instituts für Musikforschung Preußischer Kulturbesitz 2015 (2017), S. 211-240.

1 Vgl. Zenon Mojzysz, „1730: Ein Jahr im Leben Johann Adolf Hasses im Spiegel zeitgenössischer Korrespondenz", in: Johann Adolf Hasse in seiner Epoche und in der Gegenwart. Studien zur Stil- und Quellenproblematik, hrsg. von Szymon Paczkowski und Alina Zórawska-Witkowska, Warschau 2002, S. 45-52, hier v. a. S. 49 f. (Studia et dissertationes Instituti Musicologiae Universitatis Varsoviensis. Seria B T. 12.), sowie Roland Dieter Schmidt-Hensel, „La musica è del Signor Hasse detto il Sassone ...". Johann Adolf Hasses, Opere serie“ der Jahre 1730 bis 1745. Quellen, Fassungen, Aufführungen, 2 Bde. Göttingen 2009, Bd. 1, S. 38-45 (Abhandlungen zur Musikgeschichte. 19). 
liefert sind. ${ }^{2}$ Bei der Untersuchung der Überlieferung Hasse'scher Opern in Dresdner Notistenabschriften ist somit zu berücksichtigen, dass hier ein Zweig der Hofmusik in den Mittelpunkt tritt, der zum Teil andere organisatorische Rahmenbedingungen aufwies als die Instrumentalmusik. Indes sind bei den Notenschreibern durchaus personelle Überschneidungen festzustellen, so dass aus der Beschäftigung mit dem der Oper zugehörigen Quellenrepertoire Präzisierungen und neue Erkenntnisse auch für die instrumentale Überlieferung zu erwarten sind.

Die Erforschung der Schreiber musikalischer Quellen hat grundsätzlich zwei Problemkreise zu bewältigen. Zum einen gilt es, die Hand ein und desselben Schreibers in unterschiedlichen Quellen $\mathrm{zu}$ individualisieren und unterschiedliche, wenngleich möglicherweise in ihrem Schriftbild ähnliche Schreiber voneinander zu unterscheiden. Zum anderen ist zu versuchen, die solcherart individualisierten und voneinander abgegrenzten, zunächst aber namenlosen Schriftbilder den in Akten oder anderen Dokumenten namentlich nachgewiesenen Notisten zuzuordnen. Setzt die Zuordnung der Schriftbilder zu Namen voraus, dass sich entweder namentlich gekennzeichnete Schreibarbeiten nachweisen lassen (was bei professionellen Notisten eher die Ausnahme denn die Regel sein dürfte, wenngleich gerade einige der Dresdner Hofnotisten zeitweise ihre Initialen in die Schlussschnörkel ihrer Schreibarbeiten integrierten ${ }^{3}$ ) oder aber in Briefen oder Akten eigenschriftliche Nachweise der jeweiligen Personen zu finden sind, die zumindest punktuell mit der Textschrift in den Musikhandschriften übereinstimmen, so ist für die Individualisierung verschiedener Schriftbilder vor allem die Frage von Stabilität und Varianz einer Schreiberhand im Auge zu halten.

Im Allgemeinen ist davon auszugehen, dass die ausgereiften Handschriften professioneller Notisten in sich mehr oder weniger stabil sind, die Schriftformen innerhalb einer Handschrift also allenfalls geringfügig variieren und häufig auch über längere Zeiträume im Wesentlichen konstant bleiben, wohingegen bei noch ungeübten Schreibern am Anfang ihrer Notistentätigkeit und bei Musikern, die nur sporadisch für Kopierarbeiten herangezogen wurden, mit einer größeren

2 Dieser Befund steht in deutlichem Kontrast zur Überlieferung außerhalb Dresdens, wo unter Hasses Namen zahlreiche Flötenkonzerte und -sonaten in handschriftlichen Quellen oder Druckausgaben überliefert sind (vgl. Übersicht in $\mathrm{MGG}^{2}$, Personenteil Bd. 8, Sp. $812 \mathrm{f}$.); indes ist davon auszugehen, dass es sich hierbei zu einem erheblichen Teil um Fehlzuschreibungen handelt. Auffällig ist in jedem Fall, dass bislang überhaupt nur eine (teil-)autographe Quelle zu einem Instrumentalwerk Hasses bekannt ist (A-Wn: 19321: Wiener Abschrift von 6 Violinsonaten, wohl aus den frühen 1760er Jahren; auf dem Titelblatt Autorenangabe des Kopisten von Hasse durchgestrichen und eigenhändig wiederholt, außerdem einige eigenhändige Ergänzungen und Korrekturen im Notentext).

3 Dies gilt u. a. für frühe Schreibarbeiten sowohl von „Schreiber A“ alias Johann Gottfried Grundig als auch von „Schreiber D“ alias Johann Gottlieb Morgenstern; vgl. Manfred Fechner, Studien zur Dresdner Überlieferung von Instrumentalkonzerten deutscher Komponisten des 18. Jahrhunderts, Laaber 1999 (Dresdner Studien zur Musikwissenschaft. 2), S. 81 („Schreiber A“: „IGG“) bzw. 107 („Schreiber D“: „m“ bzw. „IGM“; in beiden Fällen Identifizierung in Erwägung gezogen, aber nicht für eindeutig erachtet), und Ortrun Landmann, Über das Musikerbe der Sächsischen Staatskapelle. Drei Studien zur Geschichte der Dresdner Hofkapelle und Hofoper anhand ihrer Quellenüberlieferung in der SLUB Dresden, ${ }^{2} 2010$, S. 148 (Grundig) bzw. 165f. (Morgenstern), http://nbnresolving.de/urn:nbn:de:bsz:14-qucosa-38515. Ebenso sind von Matthäus Schlettner vereinzelt mit Monogramm signierte Abschriften nachweisbar; vgl. ebd. S. 171 und 472, Abb. III.163. 
Uneinheitlichkeit bisweilen auch innerhalb ein und derselben Quelle zu rechnen ist. Im Einzelfall kann aber auch bei ausgeschriebenen Notistenschriften die konkrete Ausprägung der einzelnen Zeichen bei gleichbleibenden Grundformen durchaus eine erhebliche Schwankungsbreite aufweisen; ferner kann die prinzipielle Stabilität einer Handschrift überlagert werden durch Varianzen, die durch äußere Faktoren wie beispielsweise Unterschiede in der Papierqualität, der Größe der Notensysteme oder der Stärke der verwendeten Feder bedingt sein können. Hiervon zu unterscheiden sind Entwicklungen und Transformationen, denen eine Handschrift im Laufe der Zeit unterliegt, wobei die Umstellung auf neue Zeichenformen sozusagen ,auf einen Schlag' erfolgen kann oder in einer längeren Übergangsphase, während der alte und neue Zeichenformen nebeneinander begegnen. Umgekehrt ist zu berücksichtigen, dass bei Notisten, die über lange Jahre an demselben Hof bzw. in derselben Notistenwerkstatt arbeiten, mitunter Assimilationsprozesse zu beobachten sind, die dazu führen, dass sich die Schriftzüge der betreffenden Notisten annähern und heute bisweilen nur noch schwer voneinander zu unterscheiden sind; auch in Dresden etabliert sich spätestens um die Mitte des 18. Jahrhunderts ein typisch Dresdner Schriftstil, dem die meisten im Umkreis des Hofes tätigen Notisten verpflichtet sind und der in seinen wesentlichen Zügen bis in das erste Viertel des 19. Jahrhunderts fortwirkt. ${ }^{4}$

Vor diesem Hintergrund ergibt sich somit zum einen die Notwendigkeit, für jeden Schreiber Kriterien zu erarbeiten, anhand derer seine Schrift eindeutig zu erkennen und von anderen zu unterscheiden ist. Zum anderen kommt der chronologischen Einordnung der Schriftbelege eine besondere Bedeutung zu. Gerade dies macht Abschriften von Opern und Oratorien zu einem dankbaren Forschungsobjekt, ist doch hier im Gegensatz zu den meisten Instrumentalwerken in aller Regel das Erstaufführungsdatum und damit auch die Entstehungszeit des Werkes bekannt, was zumindest einen Terminus post quem für dessen abschriftliche Quellen definiert - in bestimmten Konstellationen aber auch eine recht genaue zeitliche Eingrenzung einzelner Quellen erlaubt.

Die Überlieferung der Opere serie, die Johann Adolf Hasse zwischen 1731 und 1756 für den sächsischen Hof komponierte, ist ungewöhnlich dicht. Bislang konnten allein aus den Federn der am Dresdner Hof tätigen Notisten rund 200 Abschriften dieser Werke nachgewiesen werden, die heute in zahlreichen Bibliotheken weltweit verwahrt werden; die tatsächliche Zahl dürfte jedoch noch deutlich höher liegen, da einstweilen bei Weitem nicht alle Quellen zu den Opern der späten 1740er und der 1750er Jahre in die Untersuchung einbezogen werden konnten. ${ }^{5}$ Herstellungszweck, Auftraggeber und Provenienzgang der Quellen bzw. Quellengruppen sind heute in vielen Fällen nicht mehr zu rekonstruieren. Umso wichtiger sind gerade im Hinblick auf Schreiberfragen diejenigen Quellen, deren ursprünglicher Entstehungszusammenhang bekannt ist oder erschlossen werden kann und für die somit der Zeitpunkt ihrer Anfertigung näher einzugrenzen ist.

4 Vgl. Annegret Rosenmüller, Die Überlieferung der Clavierkonzerte in der Königlichen Privatmusikaliensammlung zu Dresden im letzten Drittel des 18. Jahrhunderts, Eisenach 2002, S. 45 f. (Schriften zur Mitteldeutschen Musikgeschichte. 5).

5 Hingegen wurden die Quellen der Opern Hasses der Jahre 1730 bis 1745/ 51 in der Dissertation des Verfassers (vgl. Anm. 1) größtenteils berücksichtigt. 
In diesem Zusammenhang sind zunächst einige Autographen zu nennen, in denen Notisten einzelne aus einer früheren Fassung oder einem früheren Werk übernommene Sätze kopiert oder die Textunterlegung im Rezitativ eingetragen haben. ${ }^{6}$ Ferner haben sich für zehn Opern, ${ }^{7}$ außerdem für drei Oratorien ${ }^{8}$ und einige Kirchenwerke die Orchestermaterialien der Dresdner Erstaufführungen in den Beständen der SLUB Dresden erhalten. ${ }^{9}$ Zum großen Teil sind diese Orchestermaterialien heute mit braunen Kalbslederbänden ausgestattet, deren farblich voneinander abgesetzte Flächen auf Vorder- und Rückendeckel den Eindruck von Intarsien-Arbeit vermitteln. Es beweist, dass diese Orchestermaterialien nach ihrer praktischen Verwendung in die Obhut der Königin und Kurfürstin Maria Josepha gelangten, die offenbar eine Art Oberaufsicht über den Opernbetrieb führte,$^{10}$ denn für deren Bibliothek wurden sie in dieser Weise gebunden. ${ }^{11}$

Vielfach die gleichen Einbände weist auch eine fast vollständige Serie von Partiturabschriften der Dresdner Opern und Oratorien Hasses auf, die heute auf die Universitätsbibliotheken Leipzig und Halle verteilt sind. Hierbei handelt es sich offensichtlich um das Gros derjenigen Bände, die 1853 als vermeintliche Dubletten aus der Königlichen Privatmusikaliensammlung an das damalige Königliche Konservatorium in Leipzig abgegeben und von diesem in den 1920er Jahren weiterveräußert wurden. ${ }^{12}$ In einigen dieser Partituren begegnen von der Hand Hasses oder des

6 Ciro riconosciuto Karneval 1751 (I-Mc: Part. Tr. ms. 161; mit Kremmler); Ipermestra Oktober 1751 (I-Mc: Part. Tr. ms. 167; mit Kremmler und AnonH1b); Adriano in Siria Karneval 1752 (I-Mc: Part. Tr. ms. 176; mit AnonH1b); Solimano Karneval 1753 (I-Mc: Part. Tr. ms. 175; mit Grundig und AnonH1b); Artemisia Karneval 1754 (mit Kremmler und Balch); Olimpiade Karneval 1756 (mit Schlettner, Kremmler und einem weiteren Schreiber).

7 Cleofide September 1731 (Mus.2477-F-9a), Cajo Fabricio Juli 1734 (Mus.2477-F-11a), Irene Karneval 1738 (Mus.2477-F-24a), Demetrio Karneval 1740 (Mus.2477-F-12a), Artaserse September 1740 (Mus.2477-F-2a), Numa Oktober 1741 (Mus.2477-F-28a), Lucio Papirio Karneval 1742 (Mus.2477-F-32a), Didone abbandonata Oktober 1742 / Karneval 1743 (Mus.2477-F-35a), Il natal di Giove Oktober 1749 (Mus.2477-F-58a/b) und Ezio Karneval 1755 (Mus.2477-F-7a).

8 Le virtù appiè della croce 1737 (Mus.2477-D-12a), La caduta di Gerico 1744 (Mus.2477-D-18a), La deposizione dalla croce 1745 (Mus.2477-D-19a).

9 Zumindest im Falle der Opern ist davon auszugehen, dass die erhaltenen Stimmenmaterialien zur jeweiligen Erstaufführung angefertigt wurden, da die betreffenden Werke entweder in Dresden nur eine Aufführung bzw. Aufführungsserie erlebten oder der Notentext für eine etwaige Wiederaufnahme nicht oder nur unwesentlich verändert wurde (bei Didone abbandonata wurde der für 1743 überarbeitete Schluss der Oper nachträglich in das Material der Erstaufführung von 1742 eingearbeitet). Lediglich im Falle von Leucippo (1747 / 1751) und Arminio (1745/53) erfuhr eine für Dresden bzw. Hubertusburg komponierte Oper für eine spätere Wiederaufführung in Dresden eine einschneidende Überarbeitung; die Aufführungsmaterialien dieser beiden Werke sind jedoch nicht erhalten. Unübersichtlicher gestaltet sich die Sachlage hingegen bei Hasses Oratorien und kirchenmusikalischen Werken, die häufig mehrfach aufgeführt wurden und dabei bisweilen leichte Überarbeitungen erfuhren; vgl. zu den Oratorien die Ausführungen bei Michael Koch, Die Oratorien Johann Adolf Hasses. Überlieferung und Struktur, Pfaffenweiler 1989 (Musikwissenschaftliche Studien. 14).

10 Vgl. Ortrun Landmann, Katalog der Dresdener Hasse-Musikhandschriften. Die handschriftlich überlieferten Kompositionen von Johann Adolf Hasse (1699-1783) in der Sächsischen Landesbibliothek - Staats- und Universitätsbibliothek Dresden (CD-ROM-Ausgabe mit Begleitband), München 1999, S. 15 (bzw. Landmann, Drei Studien ${ }^{2} 2010$ [wie Anm. 3], S. 13f.), sowie Panja Mücke, Johann Adolf Hasses Dresdner Opern im Kontext der Hofkultur, Laaber 2003, S. $62 \mathrm{f}$.

11 Vgl. Landmann, Drei Studien 2010 (wie Anm. 3), S. 34 f., 40 f. sowie 247-253, Abb. I. 49-54.

12 Erworben wurden die Bände damals nicht direkt von den Universitäten, sondern - wie Stempel in den Bänden belegen - von der „Vereinigung der Freunde der Leipziger Universität“ bzw. der „Gesellschaft der Freunde der Universität Halle-Wittenberg“ für das jeweilige Musikwissenschaftliche Institut. Einzelne Bände der einstigen 
Notisten nachträgliche, kompositorisch relevante Änderungen einzelner Takte oder Passagen, die sich zumindest in einigen Fällen auch in den Autographen bzw. den Aufführungsmaterialien wiederfinden. ${ }^{13}$ Angesichts dieser Korrekturen sowie der ,Intarsien-Einbände ist zu vermuten, dass es sich hierbei um Abschriften handelt, die sich die Königin noch während der Probenphase anfertigen ließ, und dass die Entstehungszeit dieser Partituren mithin in das direkte zeitliche Umfeld der Erstaufführung fällt.

Ebenfalls zeitlich recht nah an der jeweiligen Erstaufführung stehen vermutlich alle oder doch die meisten Bände einer weitgehend vollständigen Serie der Dresdner Opern Hasses seit Mitte der 1740er Jahre, die heute überwiegend zum Archiv der Sing-Akademie zu Berlin gehören, das sich als Depositum in der Musikabteilung der Staatsbibliothek zu Berlin befindet, und die ergänzt werden durch zwei Partituren im genuinen Bestand der Berliner Staatsbibliothek. ${ }^{14}$ Einige dieser Partituren weisen Eintragungen auf, die von der Verwendung der Bände zur Vorbereitung der jeweiligen Berliner Aufführungen zeugen; in drei Fällen wurde die Dresdner Partitur in Berlin physisch als Direktionspartitur eingerichtet. ${ }^{15}$ Somit ist anzunehmen, dass es sich hierbei um diejenigen Partituren handelt, die sich der preußische König Friedrich II. - bekanntlich ein großer Verehrer Hasses - aus Dresden kommen ließ. Dies allein würde freilich wenig über die Entstehungszeit der Quellen aussagen, da die meisten Berliner Aufführungen Hasse'scher Opern in die Zeit nach dem Siebenjährigen Krieg fallen und somit mitunter Jahrzehnte zwischen den Dresdner und den Berliner Aufführungen liegen. ${ }^{16}$ Zumindest in vier Fällen haben sich aber in den Berliner Hofakten Zahlungsvermerke erhalten, die belegen, dass die betreffenden Opern schon wenige Monate nach der Dresdner Premiere nach Berlin gelangten, ${ }^{17}$ so dass zu vermuten ist, dass dies insgesamt die Regel war. Außerdem kann für die im Archiv der Sing-Akademie erhaltene Armi-

Konservatoriums-Sammlung wurden zu einem unbekannten Zeitpunkt abgespalten und befinden sich heute in Berlin (D-B: Mus.ms. 25030 Euristeo; venezianische Abschrift, aber mit Arienverzeichnis des Dresdner Notisten Kremmler) und möglicherweise in Washington (US-Wc: ML31.H43a\# Cajo Fabricio; die Zugehörigkeit dieser Partitur zu der Sammlung ist nicht gesichert); der Verbleib einiger weiterer einst in Leipzig nachweisbarer Bände ist heute unbekannt. Zur Geschichte und Bedeutung dieser Sammlung vgl. Maren Goltz, ,„... dass es vor Einseitigkeit der Bildung und Geschmacksrichtung bewahrt .... Zur Geschichte der Hochschulbibliothek“, in: MT-Journal. Zeitschrift der Hochschule für Musik und Theater, Felix Mendelssohn Bartholdy' Leipzig, 13 (2002), Beilage, S. 2-4, sowie Schmidt-Hensel, Hasse 2009 (wie Anm. 1), Bd. 1, S. 155-157.

13 Vgl. hierzu Schmidt-Hensel, Hasse 2009 (wie Anm. 1), Bd. 1, S. 156 f.

14 D-B: Mus.ms. 9541 / 1 Cleofide und Mus.ms. 9565/ 1 Artemisia (beide in Berlin zur Direktionspartitur der dortigen Aufführungen bearbeitet).

15 Neben den beiden in Anm. 14 genannten Partituren auch D-Bsa: SA 1090 L'eroe cinese.

$16 \mathrm{Zu}$ den Aufführungen Hasse'scher Opere serie in Berlin vgl. Schmidt-Hensel, Hasse 2009 (wie Anm. 1), Bd. 1, S. 355-364, sowie ders., „Hasses Opern auf Friedrichs Bühne. Zur Rezeption der Opere serie Johann Adolf Hasses im Berlin des 18. Jahrhunderts“, in: Johann Adolf Hasse. Tradition, Rezeption, Gegenwart. Bericht über das Symposium vom 23. bis 25. April 2010 in der Hochschule für Musik und Theater Hamburg, hrsg. von Wolfgang Hochstein, Stuttgart 2013, S. 49-68 (Hasse-Studien, Sonderband 3).

17 Vgl. die Belege bei Christoph Henzel, „Die Schatulle Friedrichs II. von Preußen und die Hofmusik (Teil 2)“, in: Jahrbuch des Staatlichen Instituts für Musikforschung Preußischer Kulturbesitz 2000, S. 175-209, hier S. 196 (Nr. 37: Mai 1750, für Attilio Regolo, Erstaufführung 12.1.1750), S. 197 (Nr. 49: April 1752, für Adriano in Siria, Erstaufführung 17. 1. 1752) und S. 200 (Nr. 76 und 78: Februar bzw. April 1756, für in Berlin angefertigte Kopien von Il re pastore, Erstaufführung 7. 10. 1755, bzw. Olimpiade, Erstaufführung 16.2.1756, die demnach zu diesem Zeitpunkt schon in Berlin vorliegen mussten). 
nio-Partitur ${ }^{18}$ die Entstehungszeit aufgrund ihrer Verwendung als Vorlage für die Berliner Produktion des Winters 1746 / 47 mit Sicherheit auf die Zeit zwischen der Erstaufführung im Oktober 1745 und dem Herbst 1746 eingegrenzt werden. Ähnliches gilt im Übrigen auch für einige weitere Partituren in anderen Bibliotheken, die ebenfalls nachweislich für auswärtige Aufführungen herangezogen wurden. Zu nennen sind hier insbesondere eine Partitur von Hasses Leucippo aus dem Oktober 1747 in der Herzog-August-Bibliothek Wolfenbüttel, die als Vorlage für die Aufführung des Werkes Ende August 1748 in Salzdahlum verwendet wurde, ${ }^{19}$ sowie eine Abschrift von Ciro riconosciuto aus dem Karneval 1751 in der Württembergischen Landesbibliothek Stuttgart, die zur Direktionspartitur der Stuttgarter Aufführung im folgenden Karneval 1752 umgestaltet wurde. ${ }^{20}$

IV

Die Dresdner Notisten, die in der Überlieferung Hasse’scher Opern der Jahre 1731 bis 1756 begegnen, lassen sich grob in zwei Gruppen bzw. ,Generationen' unterteilen. Als erste ,Generation' sind vor allem Johann George Kremmler (circa 1697-1759; zur Unterscheidung von seinem gleichnamigen Enkel auch als „Kremmler I“ bezeichnet) ${ }^{21}$ und Johann Gottfried Grundig (1706?-1773; ehemals als „Schreiber A“ bezeichnet) ${ }^{22}$ zu nennen, die in enger zeitlicher Nachbarschaft zu Hasses Amtsantritt Ende 1733 ihre Anstellung als Notisten erhielten, beide aber schon einige Jahre zuvor auf Honorarbasis tätig waren. ${ }^{23}$ Hinzu kommt der lange Zeit als „Schreiber D“ bezeichnete nebenamtliche Notist, der erst vor kurzem von Ortrun Landmann mit dem Kapellbratschisten Johann Gottlieb Morgenstern (1687-1763) identifiziert wurde; ${ }^{24}$ außerdem begegnen in Kombination mit diesen drei Notisten verschiedentlich weitere Gehilfen. Mitte/Ende der 1740er sowie Anfang der 1750er Jahre treten dann eine ganze Reihe jüngerer Notisten in Erscheinung. Auf eine Eingabe von Hasse hin wurden 1754 Matthäus Schlettner (um 1713-1792) und George Christoph Balch (gest. 1785) als zusätzliche Notisten fest angestellt; ${ }^{25}$ von diesen war, wie zu zeigen sein wird, zumindest Schlettner bereits zuvor längere Zeit auf Honorarbasis tätig. Daneben sind auch in dieser, Generation' mehrere nebenamtliche Notisten nachzuweisen, darunter Johann Gottlieb Haußstädler (um 1730? - um 1800) ${ }^{26}$ sowie ein in einigen Aktenbelegen zwischen 1750 und 1756 erwähnter Johann Leonhard Putz oder Butz (Lebensdaten unbekannt), ${ }^{27}$ für den sich Hasse in der

18 D-Bsa: SA 1071.

19 D-W: Cod. Guelf. 122 Mus. Hdschr.; vgl. auch Schmidt-Hensel, Hasse 2009 (wie Anm. 1), Bd. 1, S. 365 f.

20 D-Sl: HBXVII216 a-c.

21 Vgl. Landmann, Drei Studien 2010 (wie Anm. 3), S. 154f. (aus Gründen der Übersichtlichkeit sei an dieser Stelle zu den einzelnen Notisten primär auf die Synopse der Dresdner Hofnotisten in Landmann, Drei Studien ${ }^{2} 2010$ [wie Anm. 3], S. 141-182, als Zusammenfassung des bis dahin erreichten Forschungsstandes hingewiesen, wo auch die Nachweise in den einschlägigen früheren Arbeiten von Karl Heller, Manfred Fechner und anderen aufgeführt sind).

22 Vgl. Landmann, Drei Studien 22010 (wie Anm. 3), S. 147-149.

23 Vgl. Landmann, Katalog 1999 (wie Anm. 10), S. 26 f., sowie Landmann, Drei Studien ²2010 (wie Anm. 3), S. 126 f.

24 Landmann, Drei Studien 2010 (wie Anm. 3), S. 163 ff.; eine mögliche Identifizierung von "Schreiber D“ mit Morgenstern war schon von Fechner, Studien 1999 (wie Anm. 3), S. 107 f., erwogen worden.

25 Vgl. Landmann, Drei Studien 2010 (wie Anm. 3), S. 127.

26 Vgl. Landmann, Drei Studien 22010 (wie Anm. 3), S. 150-152.

27 Nachweise bei Landmann, Drei Studien 22010 (wie Anm. 3), S. 144 f. 
erwähnten Eingabe aus dem Jahr 1754 dahingehend verwendete, dass man „dem Notisten Buz, welcher seit verschiedenen Jahren her auch mit copiren helffen, eine Survivance Decret auf die nächst vacant werdende Notisten Stelle "erteilen möge. ${ }^{28}$

In den Cleofide-Stimmen, die im August/September 1731 angefertigt wurden und somit die früheste erhaltene Dresdner Hasse-Quelle darstellen, wirkt die Handschrift Kremmlers zwar relativ ungelenk und ungleichmäßig, zeigt aber bereits überwiegend ihre endgültigen Zeichenformen, darunter den ,ohrenförmigen' Bass-Schlüssel sowie eine meist mehr oder weniger winkelförmige Viertelpause ohne Anstrich von links (Abb. 1); allerdings begegnen vereinzelt auch noch Viertelpausen $\mathrm{m}$ it einem solchen Anstrich. Anhand dieses Kriteriums lassen sich im Übrigen einige Schreibarbeiten Kremmlers wie beispielsweise die von Landmann erwähnten Stimmensätze zu einem Confitebor von Antonio Lotti und einer Missa von Nicola Porpora, die noch einen quantitativ höheren Anteil von Viertelpausen mit Anstrich sowie rautenförmige statt stehende Kreuz-Vorzeichen aufweisen, eindeutig auf die Zeit vor Sommer 1731 einordnen, ohne dass einstweilen eine genaue Datierung möglich zu sein scheint. ${ }^{29}$ Schon in einer höchstwahrscheinlich auf $1732 \mathrm{zu}$

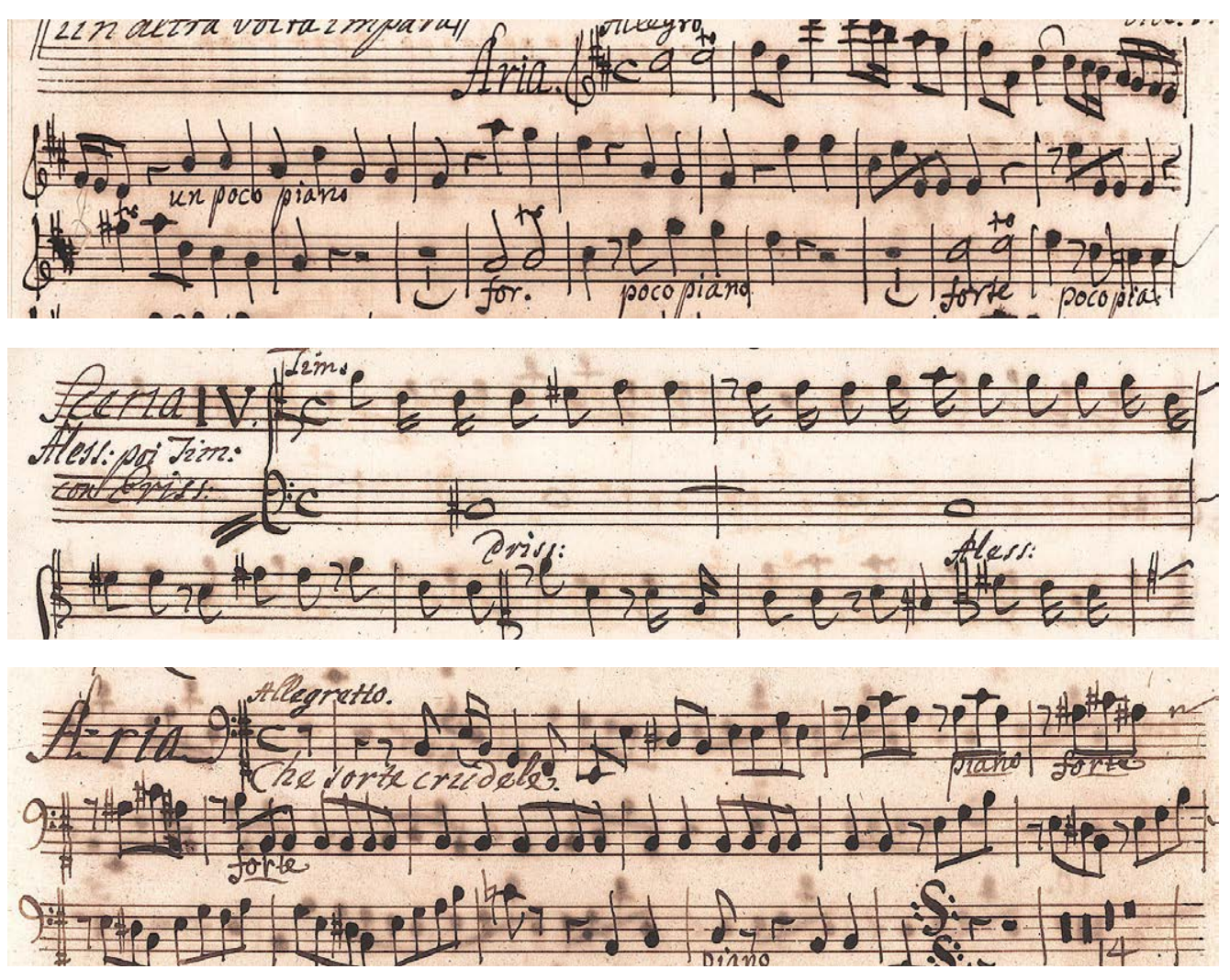

Abb. 1a-c: Johann George Kremmler, 1731 (Cleofide-Stimmen, D-Dl: Mus.2477-F-9a).

28 Zitiert nach Landmann, Drei Studien 2010 (wie Anm. 3), S. 127.

29 D-D: Mus.2159-D-11 bzw. Mus.2417-D-1, genannt bei Landmann, Drei Studien 2010 (wie Anm. 3), S. 155 (dort auf „ca. 1728“ datiert). Ob auch die bei Landmann ebenfalls für Kremmler verzeichnete zweibändige Abschrift von 1729, Recueil des XXV Concerts pour le Clavecin [...] composés par Mons: ${ }^{r}$ Chretien Pezold (Abb. III.67), von Kremmler angefertigt wurde, muss an dieser Stelle offen bleiben; wenn überhaupt, würde es sich in jedem Fall um eine höchst untypische, kalligraphische Schriftform Kremmlers handeln, die sich deutlich von den sonstigen Belegen aus dem Zeitraum um 1730 abhebt. 
datierenden Partiturabschrift von Giovanni Alberto Ristoris Oratorium La deposizione dalla croce $^{30}$ (Abb. 2a), spätestens jedoch im Sommer 1734 in den Stimmen zu Hasses Cajo Fabricio hat Kremmlers Schrift dann ihre endgültigen Zeichenformen erreicht, die sich in den folgenden Jahrzehnten bis zu seinem Ausscheiden aus dem aktiven Dienst um das Jahr 1756 herum - als letzte von Kremmler kopierte Oper Hasses konnte die im Karneval 1756 uraufgeführte Olimpiade nachgewiesen werden (Abb. 2b) - ${ }^{31}$ nicht mehr wesentlich verändern. Allerdings gehört gerade Kremmler zu den Notisten, bei denen trotz unverwechselbarem Gesamtbild und stabilen Grundformen die Ausprägung der einzelnen Zeichen durchaus erheblich schwanken kann; ferner tendieren insgesamt die Abschriften der 1750er Jahre häufig zu einem etwas größeren und vergröberten Schriftduktus.

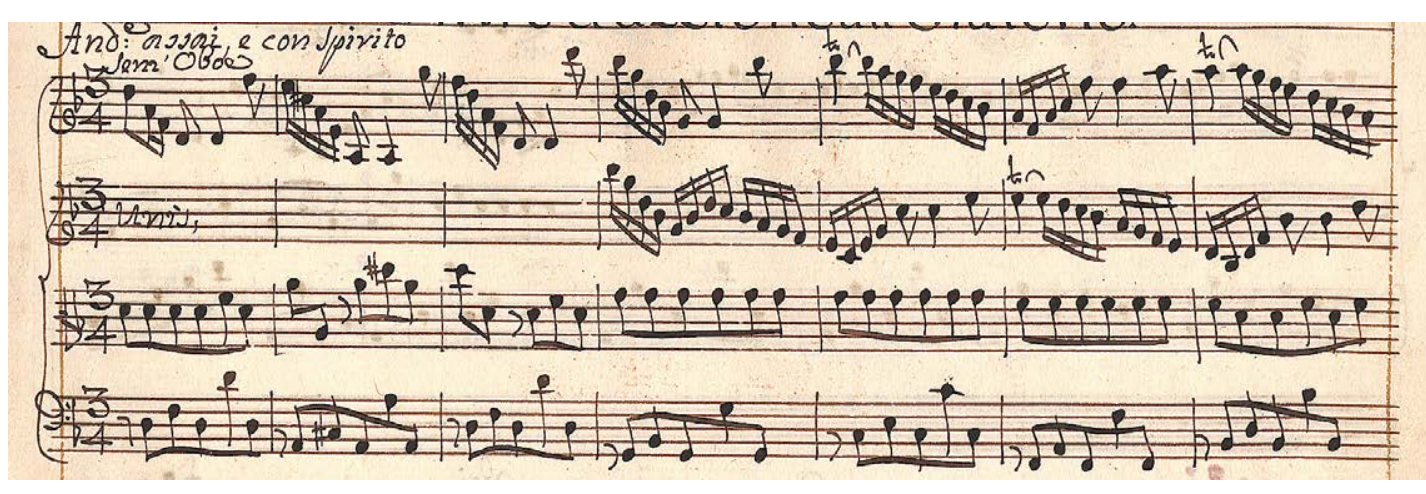

Abb. 2a: Johann George Kremmler, 1732 (Ristori, La deposizione dalla croce, D-Dl: Mus.2455-D-2).

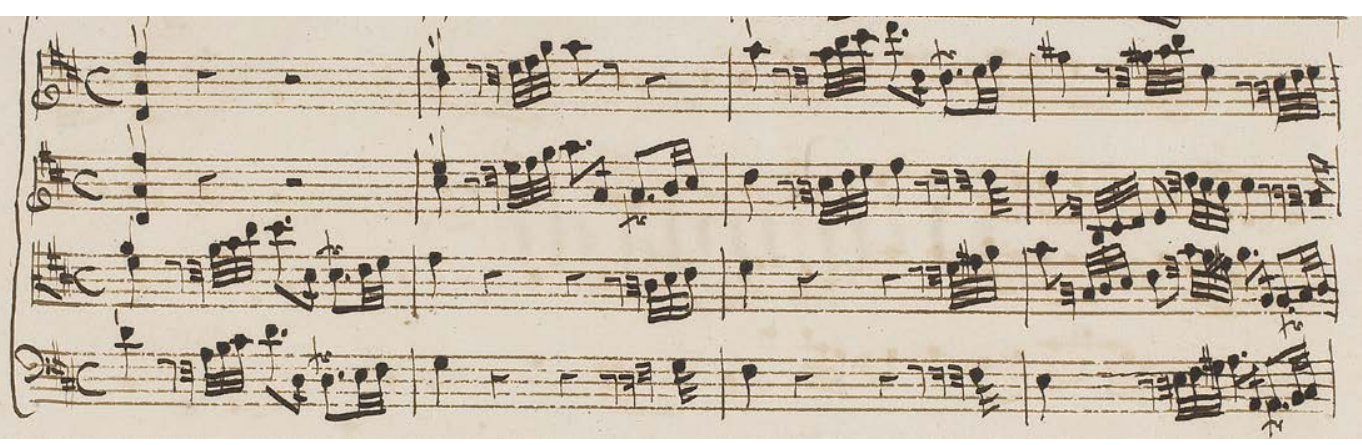

Abb. 2b: Johann George Kremmler, 1756 (L’Olimpiade, D-Bsa: SA 1077).

Für die Schrift Grundigs repräsentieren die Cleofide-Stimmen, wie bereits Manfred Fechner in seiner 1999 gedruckten Dissertation feststellte, ${ }^{32}$ ein Übergangsstadium, das sozusagen ,auf der Kippe` zwischen den von Fechner als A(1) und A(2) bezeichneten Schriftformen steht (Abb. 3). ${ }^{33}$ Der Violinschlüssel erscheint teils noch in einer frühen Form mit kurzem Abwärtsstrich, der

30 D-Dl: Mus.2455-D-2; vgl. Landmann, Drei Studien 2010 (wie Anm. 3), S. 155 und Abb. III.68.

31 D-Bsa: SA 1107.

32 Vgl. zum Folgenden Fechner, Studien 1999 (wie Anm. 3), S. 81-83 sowie S. 149-158 (Schriftproben 5a-f).

33 Einige Stimmbücher zeigen jedoch in der Sinfonia deutlich spätere Schriftformen Grundigs, was darauf hinweisen dürfte, dass hier das entsprechende Doppelblatt aus welchem Grund auch immer zu einem wesentlich späteren Zeitpunkt nachkopiert wurde. 


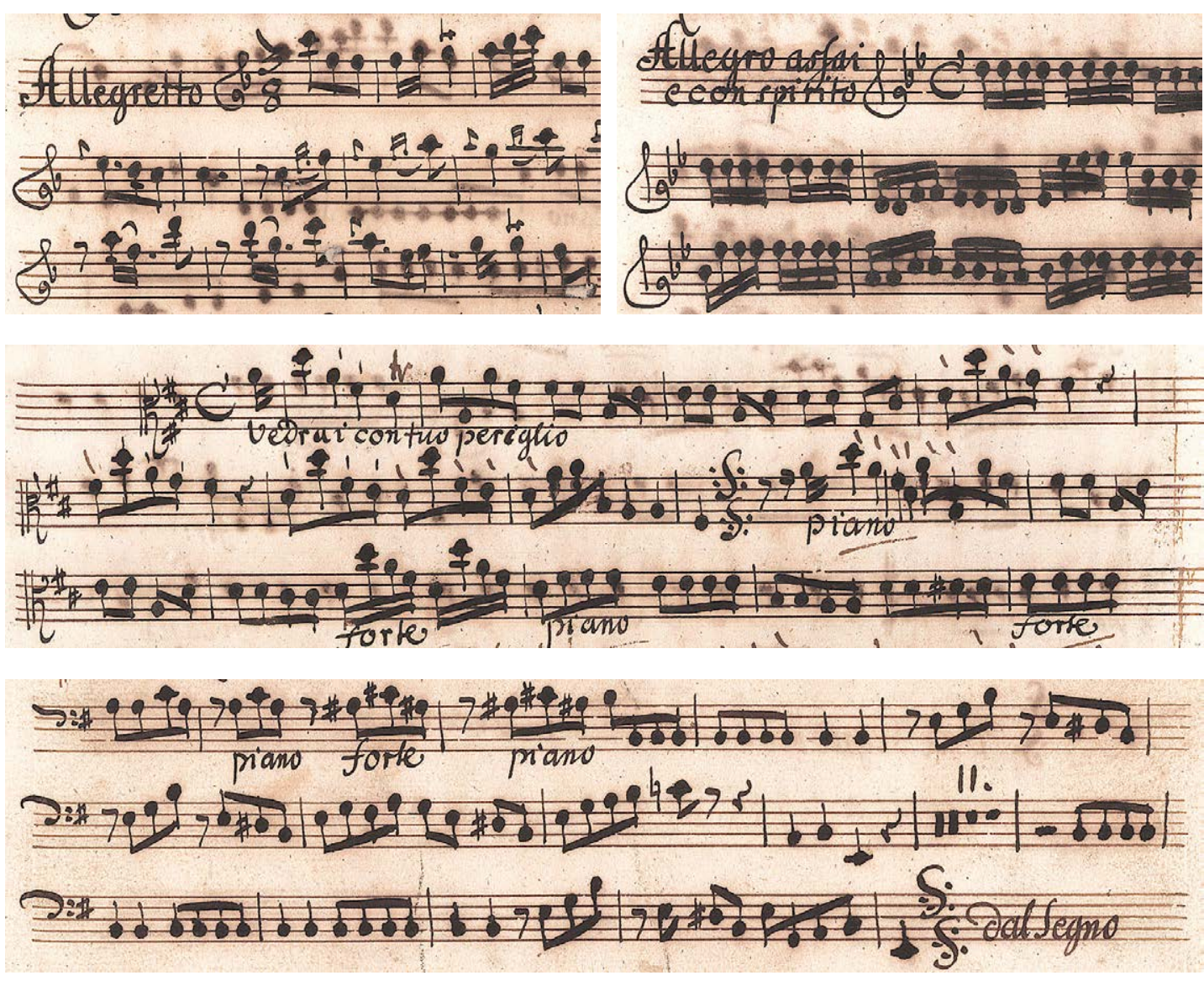

Abb. 3a-d: Johann Gottfried Grundig, 1731 (Cleofide-Stimmen, D-Dl: Mus.2477-F-9a).

gegebenenfalls direkt in das b-Vorzeichen übergeht, teils bereits mit einem bis an die unterste Linie reichenden senkrechten Strich und separaten b-Vorzeichen. Der c-Schlüssel begegnet durchweg in der ab Grundigs Schriftstadium A(2) typischen Form mit senkrechtem Doppelstrich zu Beginn des Systems und zwei bogenförmig geschwungenen Linien über und unter der c-Linie; an einigen Stellen ist jedoch beim oberen der beiden Halbbögen noch ein zweiter Verbindungsstrich zu beobachten, der wohl als Relikt der älteren, dem Schriftstadium A(1) zuzurechnenden ,Kastenform' des Schlüssels zu deuten ist. Der Bass-Schlüssel schließlich schwankt bisweilen noch zwischen der eher waagrecht liegenden Form des Schriftstadiums A(1) und der neueren, eher kreisförmig geschlossenen Variante. Knapp drei Jahre später im Cajo-Fabricio-Stimmenmaterial aus dem Frühsommer 1734 ist die Schriftentwicklung Grundigs dann weitgehend zum Abschluss gekommen (Abb. 4). Bei c- und Bass-Schlüssel haben sich nun die neuen Formen endgültig durchgesetzt, wobei der Bass-Schlüssel gegenüber 1731 deutlich stärker kreisförmig nach unten gezogen ist. Der Violinschlüssel hingegen erscheint in einer stark stilisierten Form, die hinsichtlich des Stilisierungsgrades noch wesentlich über das späteste bei Fechner genannte Schriftstadium A(4) hinausgeht. Spätere Schreibarbeiten Grundigs zeigen dann noch graduelle weitere Veränderungen (Abb. 5a-c): Der stilisierte Violinschlüssel, dessen unterer Kehrpunkt im Sommer 1734 meist etwas unter der ersten Notenlinie lag, scheint ab ungefähr 1740 in den meisten Fällen mehr oder weniger auf dieser Linie zu stehen; und während die Viertelpause 1734 und auch noch 1740 einen deutlichen Öffnungswinkel zwischen dem Anstrich von links oben und dem Aufstrich erkennen lässt, ist spätestens um 1745 / 46 kaum noch eine solche Öffnung zu sehen - wenngleich weiterhin 


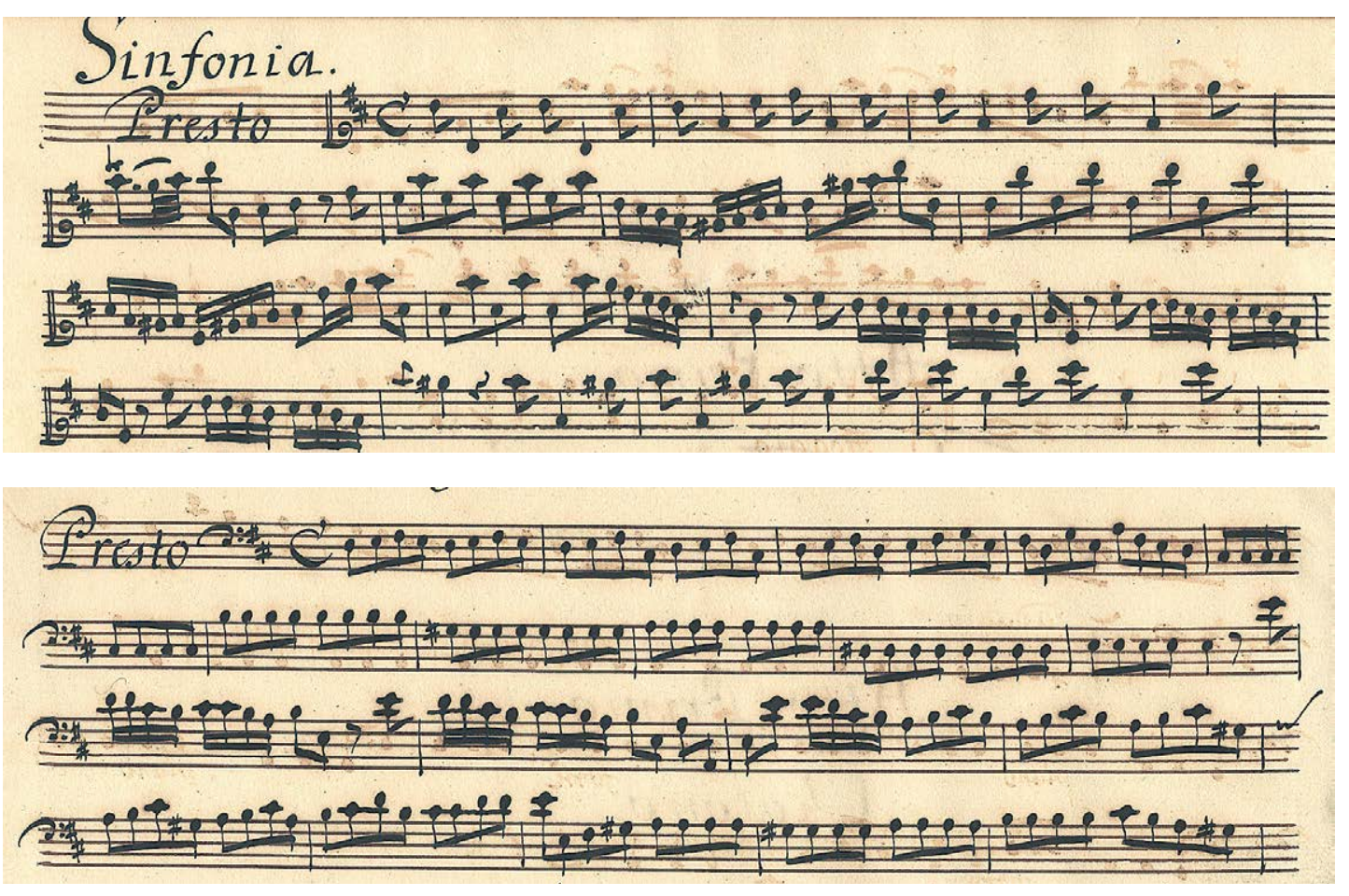

Abb. 4a-b: Johann Gottfried Grundig, 1734 (Cajo-Fabricio-Stimmen, D-Dl: Mus.2477-F-11a).

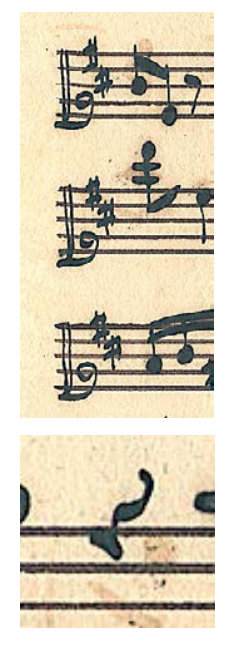

Abb. 5a: Johann Gottfried Grundig, 1734 (Cajo-Fabricio-Stimmen, wie Abb. 4).
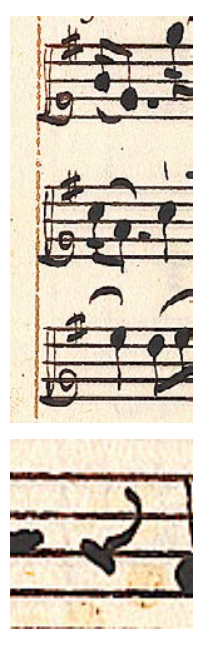

Abb. 5b: Johann

Gottfried Grundig,

1740 (Artaserse-

Stimmen, D-Dl:

Mus.2477-F-2a).
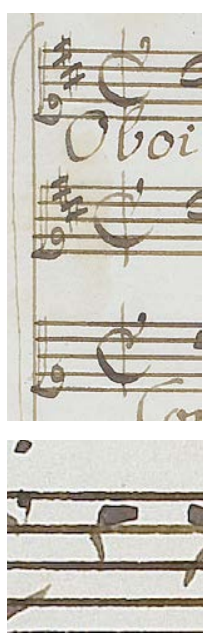

Abb. 5c: Johann Gottfried Grundig, 1745 / 46 (Arminio, D-Bsa: SA 1071 Bd. 2).

zu erkennen ist, dass Grundig anders als Kremmler die Viertelpause mit einem solchen Anstrich von links beginnt.

Prinzipiell bestätigt somit der Befund der untersuchten Quellen zu Opern Hasses die von Fechner anhand der Instrumentalüberlieferung getroffene Einschätzung, dass die Umstellung auf die endgültigen Schriftformen Grundigs um 1733/34 im Großen und Ganzen abgeschlossen war. ${ }^{34}$ Allerdings verschiebt sich das Verhältnis der von Fechner und vor ihm schon von Karl Heller $^{35}$ ausgehend vom Instrumentalrepertoire so bezeichneten „Haupt-“ und „Spätformen“ des

34 Vgl. Fechner, Studien 1999 (wie Anm. 3), S. 82 f.

35 Vgl. Karl Heller, Die deutsche Überlieferung der Instrumentalwerke Vivaldis, Leipzig 1971, S. 30-38 und 59-63 (Schriftproben 3a-e) (Beiträge zur musikwissenschaftlichen Forschung in der DDR. 2). 
einstmaligen "Schreibers A“ nach seiner Identifizierung als Grundig und im Lichte der Hasse-Überlieferung: Die einst so genannten „Haupt-Schlüsselformen“ entpuppen sich, gemessen an der gesamten Wirkungszeit Grundigs, als relativ frühe Schriftformen, wohingegen die für die Instrumentalüberlieferung als „spät“ titulierten Formen tatsächlich im Wesentlichen die Hauptschriftformen ab circa 1733/34 bis in die 1760er Jahre hinein - bzw. für den Violinschlüssel sogar erst eine Übergangsform zur endgültigen Ausprägung - darstellen. Dass die eigentlichen Hauptschriftformen Grundigs im Instrumentalrepertoire offenbar nur selten begegnen, dürfte damit zusammenhängen, dass mit dem Amtsantritt Hasses der Bedarf an Kopialien für den Bereich der Oper und des Oratoriums sprunghaft anstieg und somit die Arbeitskraft der beiden etatmäßigen Kopisten - also Kremmler, der offenbar auch zuvor kaum für Instrumentalmusik herangezogen worden war, und Grundig - weitgehend von diesen Bereichen sowie von der Kirchenmusik absorbiert wurde. ${ }^{36}$

Hiermit zusammenhängen dürfte die ganz erhebliche Schreibleistung von „Schreiber D“ alias Johann Gottlieb Morgenstern namentlich im instrumentalen Repertoire ab circa Anfang/Mitte der 1730er Jahre. In der Überlieferung Hasse'scher Opern und Oratorien begegnet dieser Schreiber hingegen deutlich seltener als Kremmler und Grundig. Seine Hand findet sich erstmals in den Stimmen zu Cajo Fabricio aus dem Sommer 1734, außerdem in den meisten erhaltenen Stimmenmaterialien aus der ersten Hälfte der 1740er Jahre; ${ }^{37}$ ferner konnten fünf Partiturabschriften ${ }^{38}$ und einige einzeln kopierte Arien aus verschiedenen Opern der 1730er und frühen 1740er Jahre nachgewiesen werden. Von besonderem Interesse für die Schriftentwicklung dieses Notisten sind die Cajo-Fabricio-Stimmen, da hier die Stilisierung des Violinschlüssels bei Morgenstern noch nicht so weit fortgeschritten ist wie gleichzeitig bei Grundig (Abb. 6a/7a). Somit wird deutlich, dass anders als bis vor einigen Jahren angenommen nicht etwa "Schreiber A“ (alias Grundig) sich bei der Umstellung seiner Schlüsselformen Anfang der 1730er Jahre an „Schreiber D“ (alias Morgenstern) orientierte, sondern dass vielmehr umgekehrt Grundig Mitte der 1730er Jahre Vorbildwirkung für Morgenstern hatte. Wann genau auch bei Morgenstern die Stilisierung des Violinschlüssels abgeschlossen war, ist anhand der Hasse-Überlieferung nicht näher einzugrenzen, da in diesem Quellenkorpus für die zweite Hälfte der 1730er Jahre einstweilen keine sicher datierbaren Schriftbelege Morgensterns nachgewiesen werden konnten. Spätestens in den Stimmen zu Artaserse aus dem Herbst 1740 hat dann aber auch Morgensterns Schrift im Wesentlichen ihre endgültige Ausprägung - das von Fechner als D(6)/(7) bezeichnete Stadium ${ }^{39}$ - erreicht, wobei die Stilisierung des Violinschlüssels nun sogar noch etwas weiter geht als bei Grundig (Abb. 6b/7b):

36 Vgl. auch Fechner, Studien 1999 (wie Anm. 3), S. 83, der darauf hinweist, dass „Schreiber A“ „nach ,um 1740“ zumindest als Notist für den hier zur Untersuchung anstehenden Repertoire-Bereich wohl kaum noch tätig war".

37 An den oben in Anm. 7 und 8 genannten Aufführungsmaterialien der Werke aus den Jahren 1740 bis 1745 war Morgenstern lediglich im Falle der Stimmen zur Karnevals-Oper des Jahres 1740 Demetrio sowie zum 1744 aufgeführten Oratorium La caduta di Gerico nicht beteiligt.

38 B-Bc: 14989 (La clemenza di Tito), D-Hs: ND VI 2936 (Demetrio), B-Bc: 2143 und I-PLcon: Pisani 35 (jeweils Numa, in I-PLcon zusammen mit Kremmler und Grundig), D-Bsa: SA 1087 (Didone abbandonata).

39 Zur Schriftentwicklung von „Schreiber D“ insgesamt vgl. Fechner, Studien 1999 (wie Anm. 3), S. 108-113 sowie S. 159-186 (Schriftproben 6a-h). 


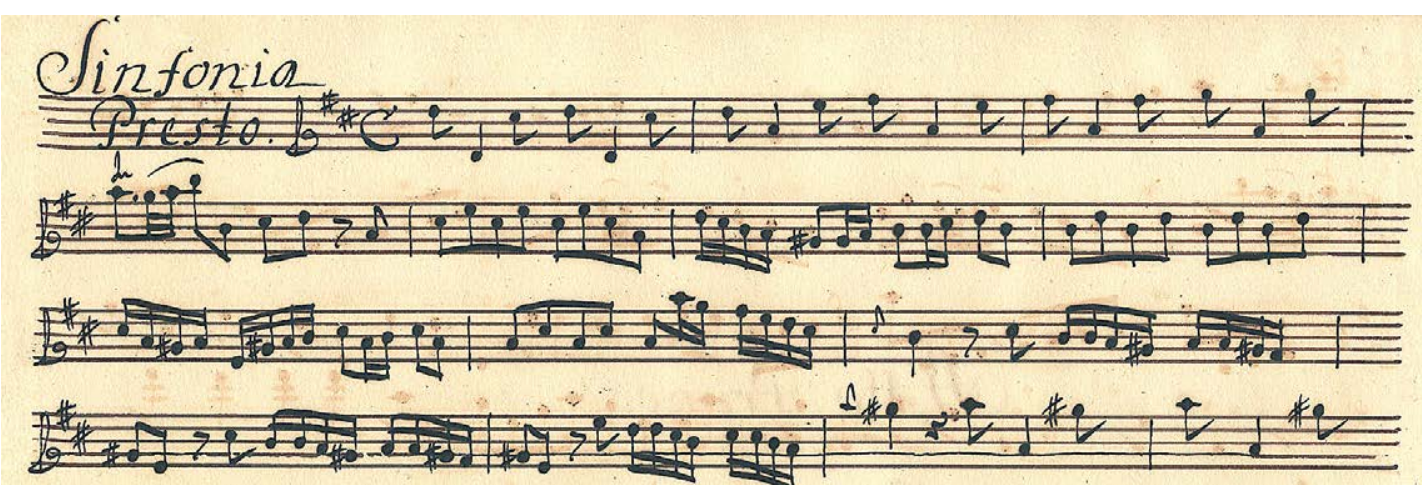

Abb. 6a: Johann Gottlieb Morgenstern („Schreiber D“), 1734 (Cajo-Fabricio-Stimmen, wie Abb. 4a-b).

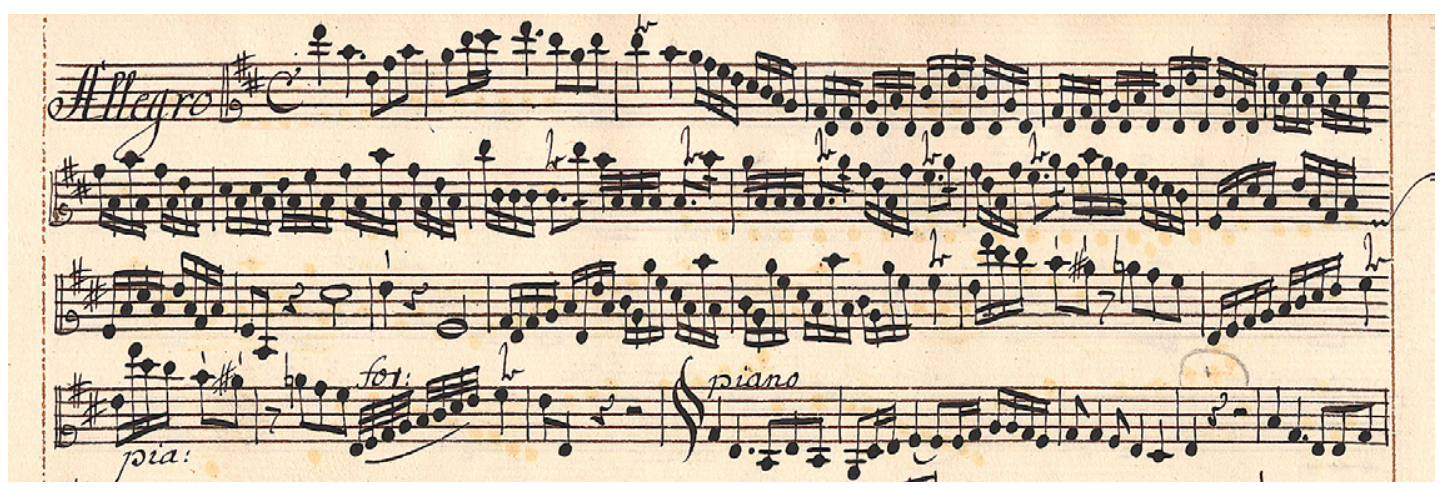

Abb. 6b: Johann Gottlieb Morgenstern („Schreiber D“), 1740 (Artaserse-Stimmen, D-Dl: Mus.2477F-2a).

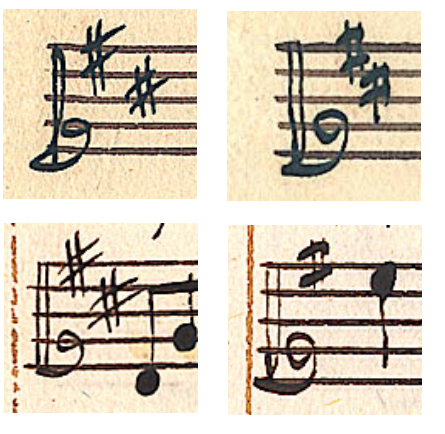

Abb. 7a-b: Violinschlüssel von Morgenstern („Schreiber D“; jeweils links) und Grundig (jeweils rechts); obere Reihe jeweils 1734, untere jeweils 1740 (Quellen wie Abb. 6a-b).

Während Grundig zu Beginn des Systems einen senkrechten Strich zieht, diesen mit einem zweiten, zunächst rechts davon parallel verlaufenden Strich im unteren Bereich berührt oder kreuzt und dann direkt aus diesem zweiten senkrechten Strich in den Bogen um die g-Linie übergeht, ist bei Morgenstern der g-Bogen separat an den auf seiner ganzen Länge parallel verlaufenden Doppelstrich angesetzt. Die späteste Oper Hasses, aus der Morgenstern einzelne Arien kopiert hat, ist nach bisherigem Kenntnisstand die Erstfassung des Dresdner Arminio aus dem Oktober $1745 .{ }^{40}$ Auch wenn das Erstaufführungsdatum hier lediglich einen Terminus post quem darstellen kann, spricht doch einiges dafür, dass „Schreiber D“ alias Morgenstern ab der zweiten Hälfte der 1740er Jahre nicht mehr für den Opernbereich herangezogen wurde; das von Fechner für die erste Hälfte der 1750er Jahre dokumentierte späteste Schriftstadium D(8) mit seinem deutlich veränderten Bass-Schlüssel jedenfalls ist in der Überlieferung Hasse’scher Opern nicht mehr zu belegen.

40 Einzelabschriften dreier Arien: D-Mbs: Mus.ms. 20 882a (1-3). 
Die Beobachtung, dass Morgenstern vermutlich nach 1745/46 nicht mehr für die Kopiatur von Opern herangezogen wurde, korreliert zeitlich ziemlich exakt mit dem Auftreten dreier neuer Notisten ab etwa 1745/46. Erstmals in der oben erwähnten Arminio-Partitur im Archiv der Sing-Akademie zu Berlin, die nachweislich aus den Jahren 1745 / 46 stammen muss, begegnet im dritten Akt ein ,neuer' Notist, der im Zusammenhang mit der Erforschung der Hasse-Handschriften der Staats- und Universitätsbibliothek Hamburg erstmals beschrieben wurde und dort das Sigel „AnonH1“ erhalten hat. ${ }^{41}$ Charakteristisch für diesen Notisten sind unter anderem die an Kremmler orientierten Grundformen des Violin- und des c-Schlüssels sowie die wie bei Kremmler ohne linken Anstrich ausgeführte Viertelpause, ferner der in dieser frühen Schriftphase "AnonH1a“ stark eingerollte Bass-Schlüssel und die gut lesbare, dabei aber in sich leicht unregelmäßige und in ihrem Duktus bisweilen etwas stockend wirkende Textschrift (Abb. 8). Um 1751 ändert sich die Form des Bass-Schlüssels grundlegend, der im späteren Schriftstadium „AnonH1b“ nicht mehr eingerollt, sondern waagrecht weit nach links ausgezogen ist und in einem Bogen nach oben ausläuft. Die Übergangsphase zwischen beiden Schriftstadien, die insbesondere durch schwankende Formgebung des Bass-Schlüssels gekennzeichnet ist, ist in einer Abschrift der Karnevalsoper des
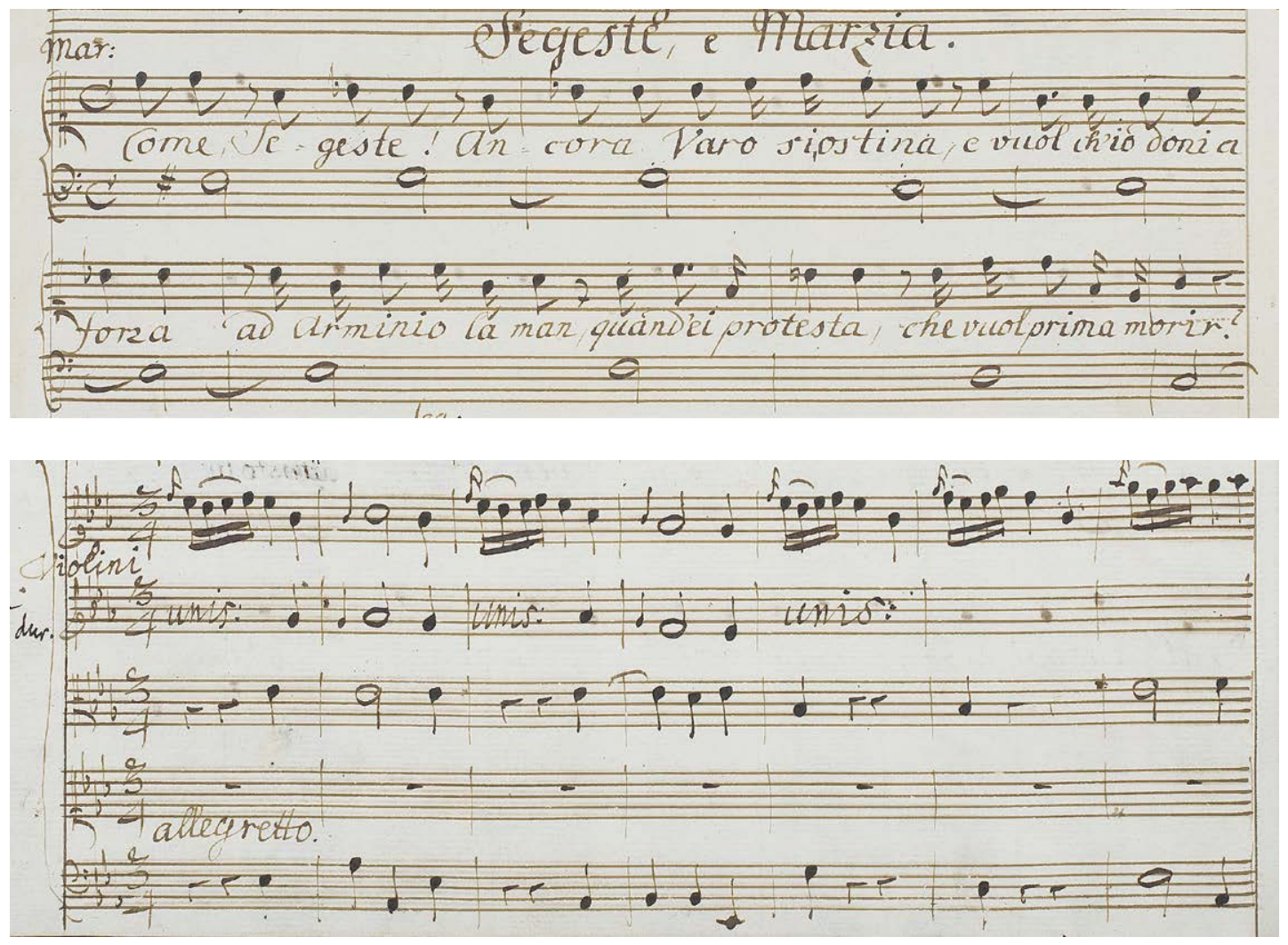

Abb. 8a-b: „AnonH1a“, 1745/46 (Arminio D-Bsa: SA 1071, Bd. 3).

41 Vgl. Roland Dieter Schmidt-Hensel, „Anmerkungen zu den Schreibern und zum Quellenwert der Hassiana in der Staats- und Universitätsbibliothek Hamburg“, in: Hasse-Studien 6, hrsg. von Wolfgang Hochstein und Reinhard Wiesend, Stuttgart 2006, S. 20-51, hier S. 21 f. und 36-39 (Abb. 5-6), sowie Schmidt-Hensel, Hasse 2009 (wie Anm. 1), Bd. 1, S. 146-148 und 469-470 (Abb. 8-9). 
Jahres 1751 Ciro riconosciuto (Abb. 9) ${ }^{42}$ sowie im Stimmenmaterial zu Hasses d-Moll-Messe, die erstmals anlässlich der Weihe der Katholischen Hofkirche im Juni 1751 erklang, dokumentiert. ${ }^{43}$ Insgesamt spielt „AnonH1“ in der Überlieferung der Opern Hasses bis hin zu Ezio aus dem Karneval 1755 eine erhebliche Rolle: Bislang konnten für ihn rund 35 Partiturabschriften nachgewiesen werden; ferner begegnet seine spätere Schriftform „AnonH1b“ auch in einigen Autographen der Jahre 1751 bis 1753 als Gehilfe Hasses. ${ }^{44}$ Inwieweit der Kopist daneben in jenen Jahren auch für die Anfertigung von Aufführungsmaterialien herangezogen wurde, ist nicht mehr festzustellen, da sich die entsprechenden Quellen zu Hasses Opern der 1750er Jahre abgesehen von den von Grundig und Balch ausgeschriebenen Stimmen zu Ezio von $1755^{45}$ nicht erhalten haben.

In der oben erwähnten, auf den Zeitraum zwischen Oktober 1747 und Sommer 1748 datierbaren Wolfenbüttler Leucippo-Abschrift begegnen dann zusammen mit „AnonH1“ zwei weitere ,neue Notisten. Der dritte Akt dieser Partitur ist trotz der zum Teil noch etwas ungelenken Text-

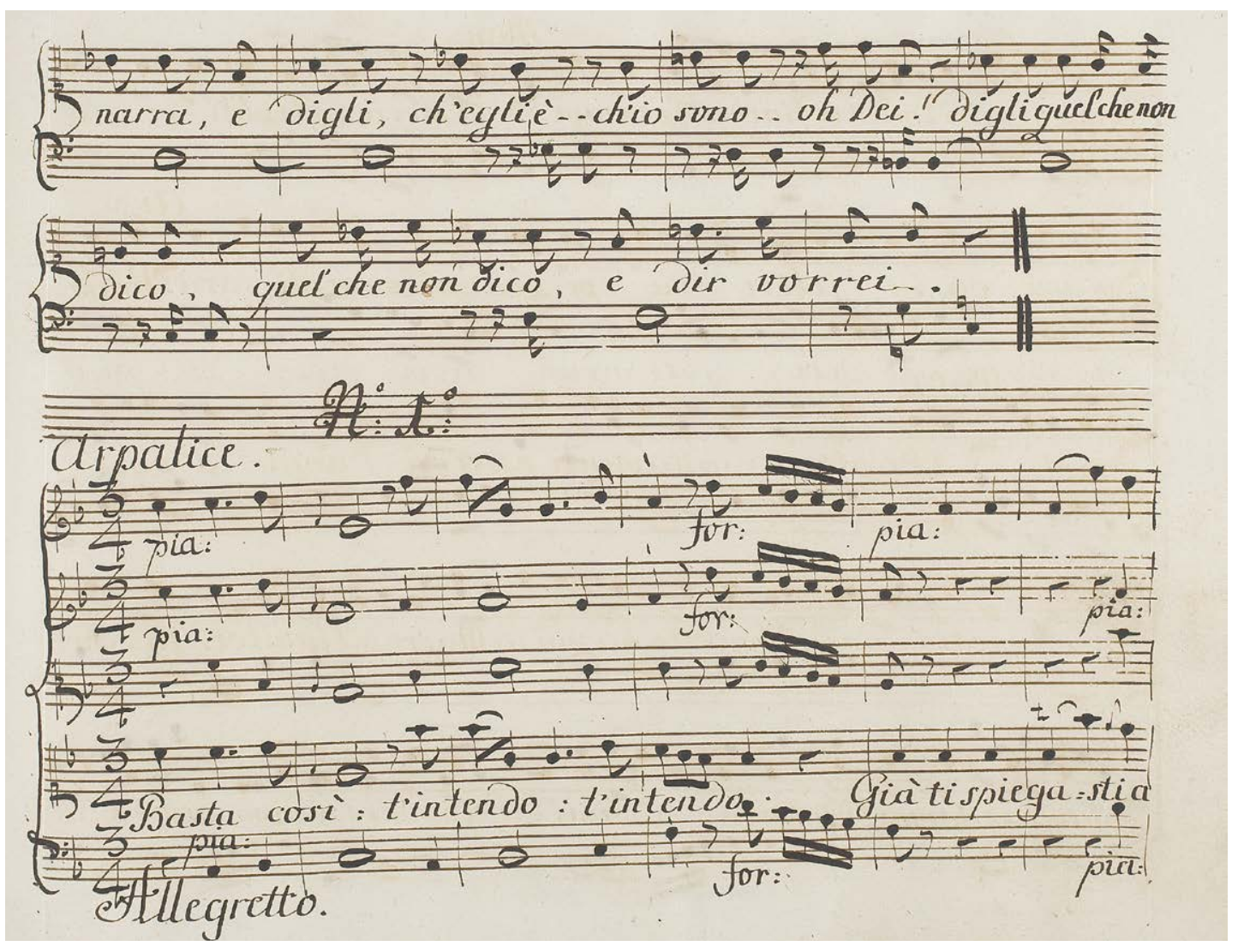

Abb. 9: „AnonH1b“, um 1751 (Ciro riconosciuto D-B: N.Mus.ms.10399).

42 D-B: N.Mus.ms.10399 (Atto I) und D-Hs: ND VI 2933a (Atto II+III; die heute auf zwei Bibliotheken verteilten Bände dürften ursprünglich zusammengehört haben); erste Anzeichen für eine Öffnung des Bass-Schlüssels sind bereits in den von „AnonH1“ geschriebenen Teilen der wohl im Frühjahr 1750 angefertigten Attilio-Regolo-Partitur D-Bsa: SA 1077 zu beobachten.

43 D-Dl: Mus.2477-D-44; die Übergangsform ist allerdings nur im Credo (-D-44,3) nachweisbar, während in allen anderen Sätzen noch die frühe Schriftform begegnet.

44 I-Mc: Part. Tr. Ms. 167 (Ipermestra, Fassung Hubertusburg, Oktober 1751), Part. Tr. Ms. 176 (Adriano in Siria, carn. 1752) und Part. Tr. Ms. 175 (Solimano, carn. 1753).

45 D-Dl: Mus.2477-F-7a. 
schrift unschwer als frühe Schreibarbeit von Matthäus Schlettner zu identifizieren, der somit seit circa 1747 / 48 in Dresden als Notenschreiber tätig gewesen sein muss und wie erwähnt im Jahr 1754 als Hofnotist fest angestellt wurde (Abb. 10). Der Schreiber des zweiten Aktes zeigt auf den ersten Blick Ähnlichkeit mit „AnonH1“; bei genauerer Betrachtung zeigen sich dann aber doch einige charakteristische Unterschiede beispielsweise bei der Form der Viertelpause mit Anstrich, bestimmten Buchstabenformen sowie dem insgesamt flüssigeren Duktus der Textschrift. Dieser ebenfalls erstmals im Zusammenhang mit den Hamburger Hassiana beschriebene $^{46}$ und dort als "AnonH2“ bezeichnete Notist (Abb. 11) dürfte höchstwahrscheinlich mit dem bei Fechner als „Schreiber T“ bezeichneten Notisten identisch sein. ${ }^{47}$ In einer offensichtlich sehr frühen Schriftphase begegnet er als alleiniger Schreiber auch in einer nicht näher datierbaren, aber demnach in jedem Fall zeitlich vor der Leucippo-Partitur einzuordnenden Abschrift von Hasses Dresdner Demetrio im Bestand der Sing-Akademie zu Berlin, die recht anschaulich zeigt, wie sich die Schriftzüge eines offensichtlich noch ungeübten Schreibers im Verlauf der drei Bände sichtbar konsolidieren und stabilisieren (Abb. 12)..$^{48}$ Die weitere Entwicklung des Schreibers
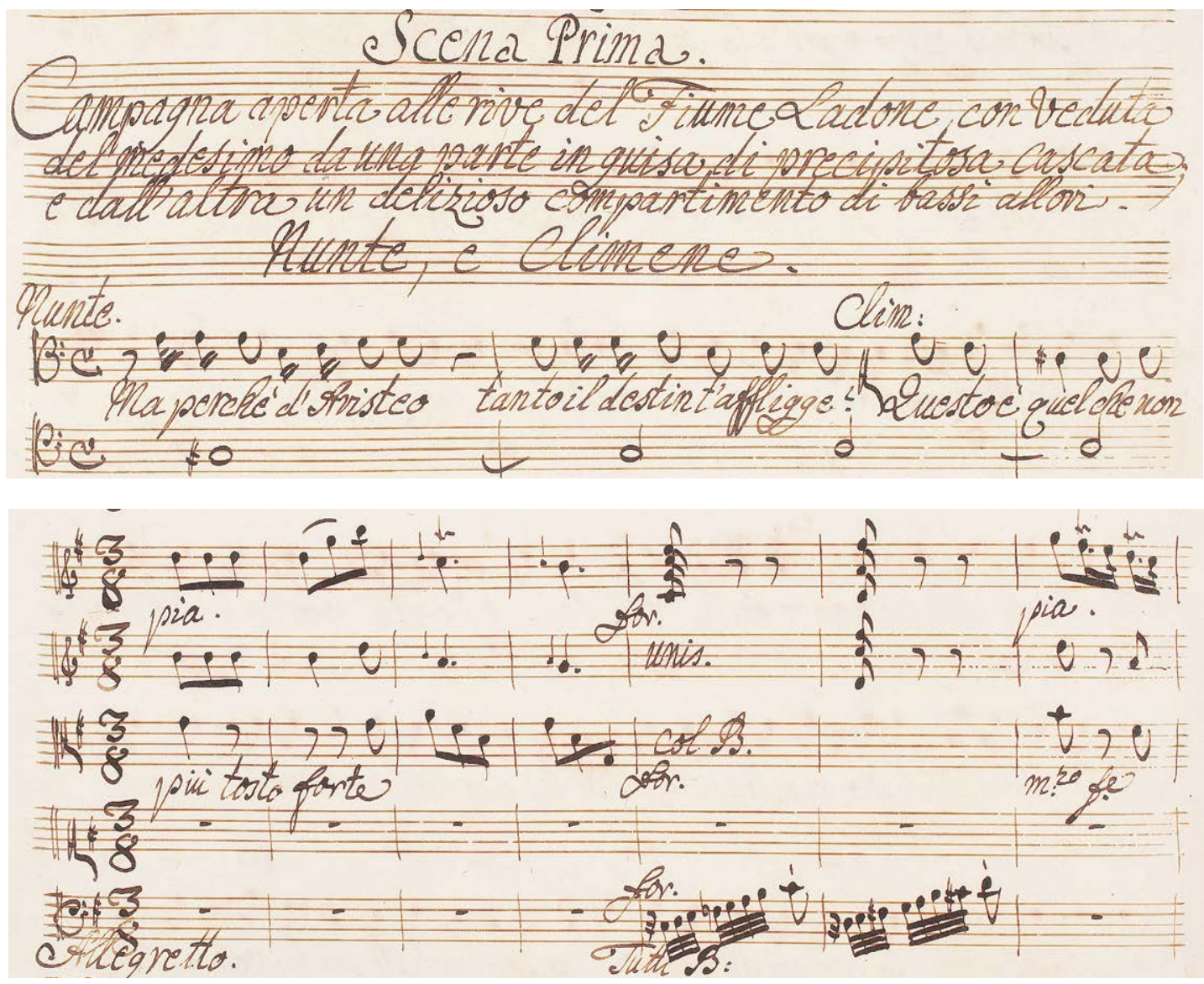

Abb. 10a-b: Matthäus Schlettner, 1747 / 48 (Leucippo D-W: Cod.Guelf. 122 Mus. Hdschr., Bd. 3).

46 Vgl. Schmidt-Hensel, Anmerkungen zu den Schreibern 2006 (wie Anm. 41), hier S. 21 f. und 40-43 (Abb. 7-8), sowie Schmidt-Hensel, Hasse 2009 (wie Anm. 1), Bd. 1, S. 146-148 und 471-472 (Abb. 10-11, identisch mit den vorgenannten).

47 Abb. bei Fechner, Studien 1999 (wie Anm. 3), S. 202.

48 D-Bsa: SA 1094. 


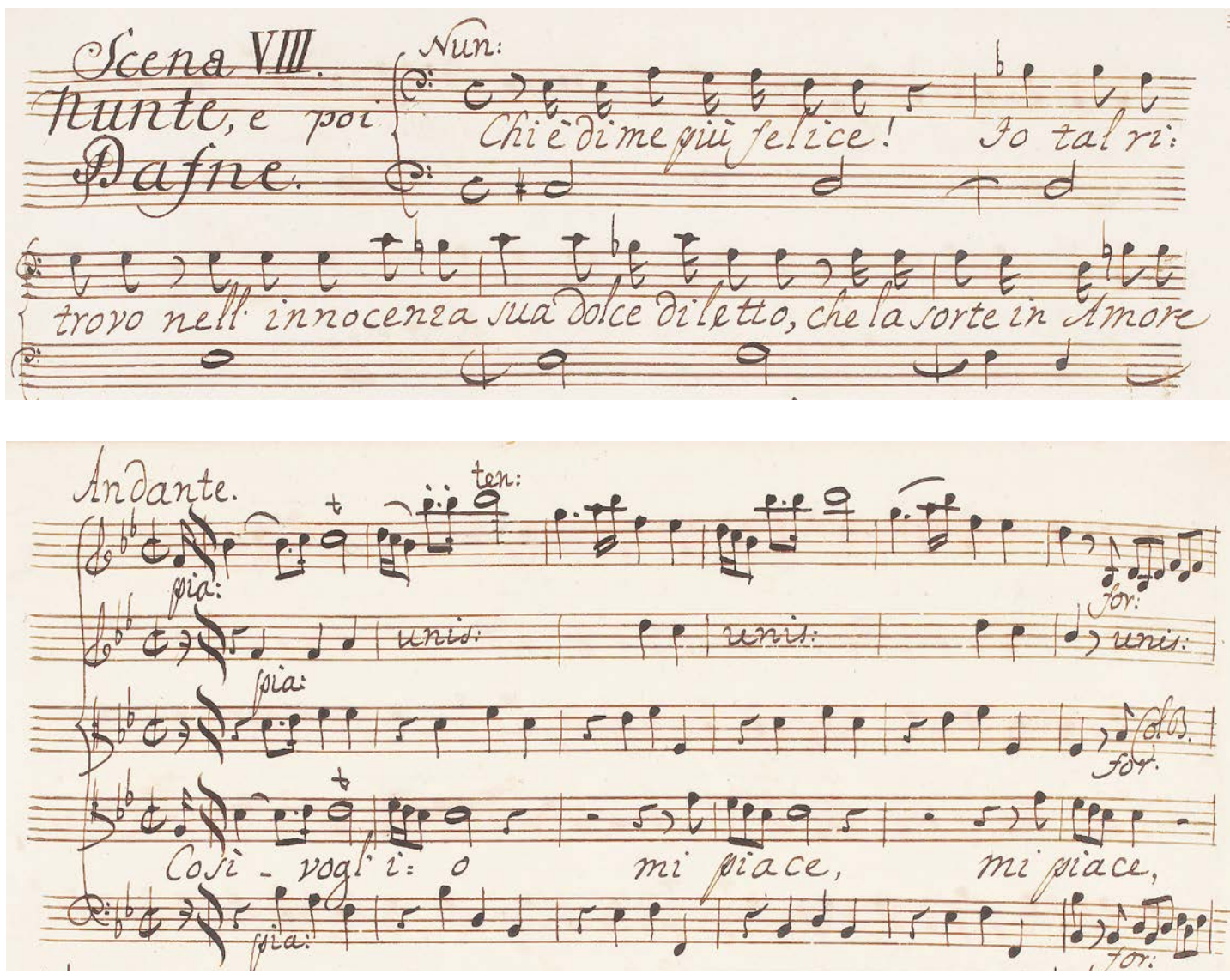

Abb. 11a-b: „AnonH2a“ (= „Schreiber T“), 1747/48 (Leucippo D-W: Cod.Guelf. 122 Mus. Hdschr., Bd. 2).
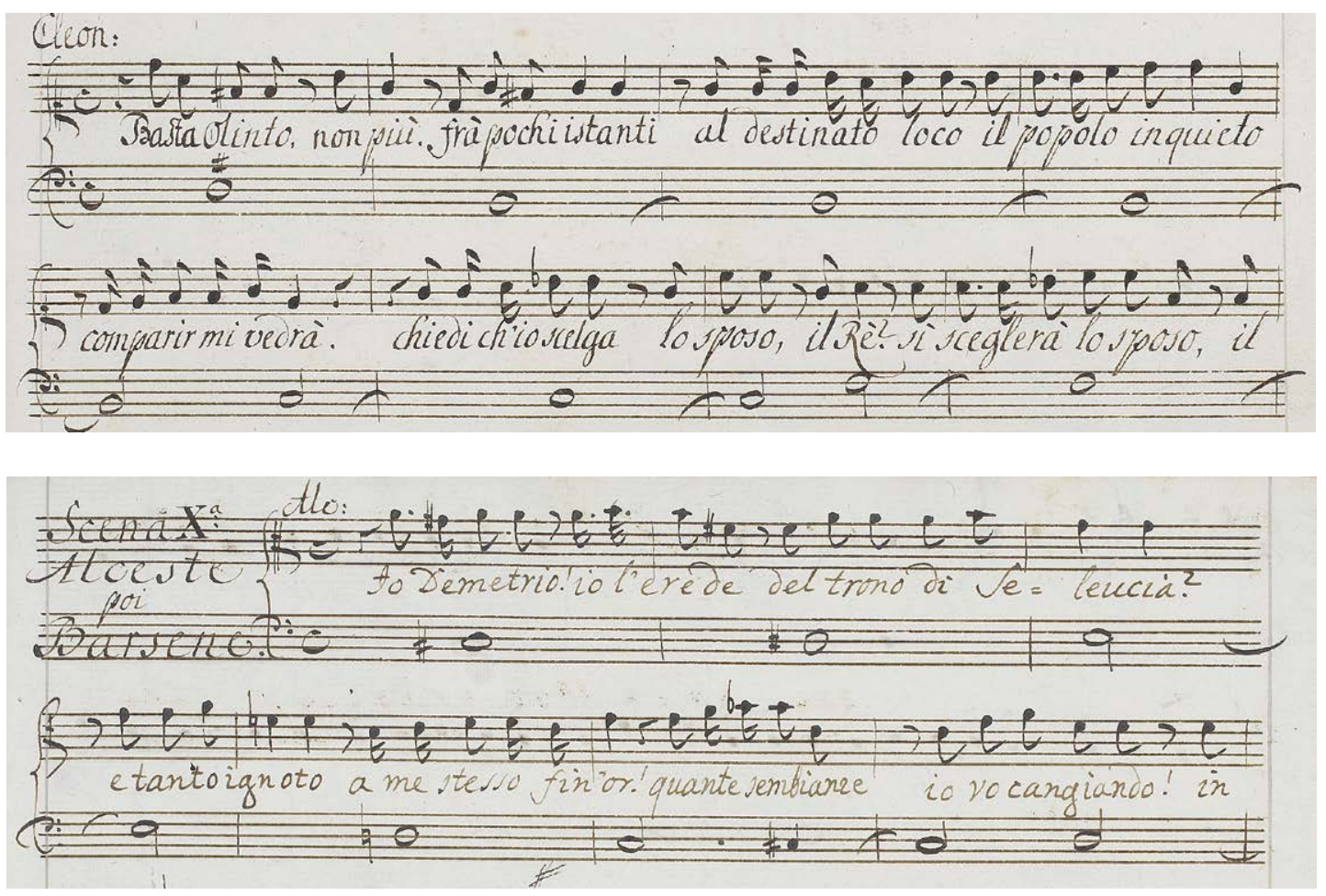

Abb. 12a-b: „AnonH2a“ (= „Schreiber T“), vor 1747 / 48 (Demetrio D-Bsa: 1077, Bd. 1 bzw. 3). 
„AnonH2“ alias „Schreiber $\mathrm{T}$ “ ist anhand der Hasse-Überlieferung nur sporadisch weiterzuverfolgen. In einer leicht abgewandelten, späteren Schriftform „AnonH2b“ begegnet er wie „AnonH1“ im Stimmenmaterial der d-Moll-Messe von 1751 (Abb. 13) ${ }^{49}$ sowie in einer wohl ungefähr in dieselbe Zeit gehörenden Attilio-Regolo-Partitur. ${ }^{50}$ In Quellen zu späteren Opern Hasses konnte „AnonH2“/ „Schreiber T“ - der wie gesagt ohnehin in diesem Repertoire nur sporadisch auftritt bislang nicht mehr nachgewiesen werden.

Für die Schriftentwicklung George Christoph Balchs, der 1754 zusammen mit Schlettner seine Bestallung als Notist erhielt und um 1765 als Kontrabassist in die Hofkapelle wechselte, aber nebenamtlich weiterhin als Notist tätig blieb, ${ }^{51}$ ergeben sich aus der Hasse-Überlieferung keine neuen Hinweise. Anders als Schlettner, der wie gezeigt bereits 1747 / 48 nachweisbar ist, stammen die frühesten sicher datierbaren Schriftbelege Balchs in diesem Quellenkorpus erst aus dem Win-
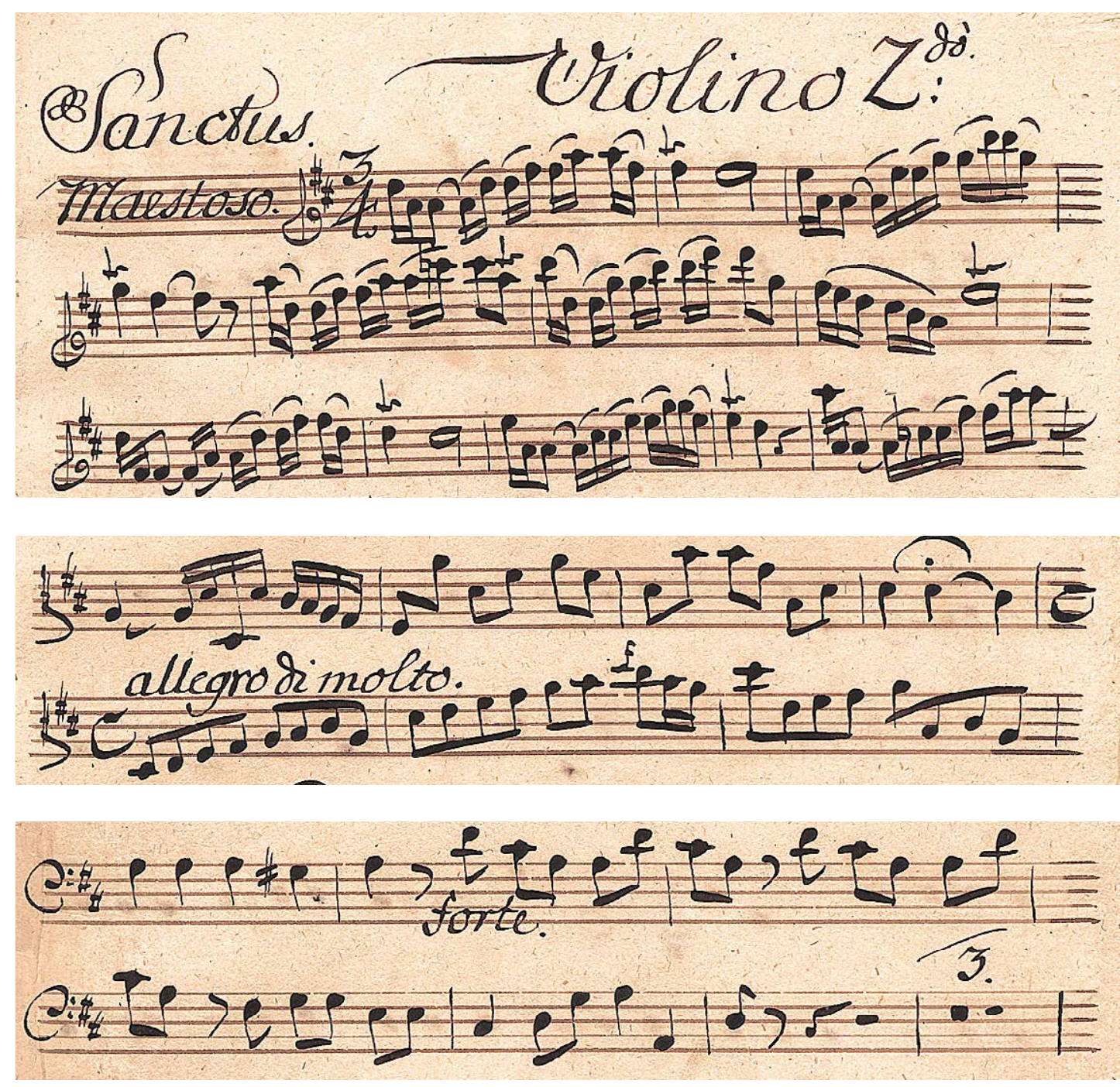

Abb. 13 a-c: „AnonH2“ (= „Schreiber T“), 1751 (Missa d-Moll Stimmen (Sanctus), D-Dl: Mus.2744-D-44,4).

49 D-Dl: Mus.2477-D-44; nur im Sanctus und Agnus Dei (-D-44,4 und -D-44,5).

50 D-Hs: ND VI 2931.

51 Zur Biographie Balchs vgl. Landmann, Drei Studien 22010 (wie Anm. 3), S. 141 f. 
ter $1753 / 54 .^{52}$ Dies schließt freilich keineswegs aus, dass er sich in den Jahren zuvor anderweitig Meriten als Notist und / oder Musiker erworben hatte..$^{53}$ Die mit Sicherheit in die mittleren 1750er Jahre zu datierenden Hasse-Abschriften Balchs ${ }^{54}$ zeigen jedenfalls keine nennenswerten Divergenzen, wie sie bei einem noch völlig unerfahrenen Schreiber zu erwarten wären. Vermutlich Anfang der 1760er Jahre scheint Balch im Übrigen zumindest vorübergehend in engerem Kontakt mit dem Leipziger Verleger Breitkopf gestanden zu haben, wie etliche von ihm angefertigte Opernabschriften mit gedruckten Titelblättern des Verlagshauses belegen. ${ }^{55}$

Mit den Notisten „AnonH1“ und „AnonH2“ (alias „Schreiber T“) treten im Zusammenhang der Überlieferung Hasse'scher Opern zwei Notisten in das Blickfeld der Forschung, die offensichtlich ab den mittleren/späten 1740er Jahren in erheblichem Maße Schreibarbeiten am Dresdner Hof geleistet haben, ohne dass sie bislang einem der aus den Akten bekannten Namen eindeutig zuzuordnen wären. Zugleich berührt die Frage nach einer möglichen Identifizierung dieser beiden Notisten die in der Literatur über die Dresdner Hofkapelle und ihre Schreiber seit längerem umstrittene Problematik nach der Identität oder Nicht-Identität aller oder einiger der von Fechner als „Schreiber O“, „S“ und „T“ differenzierten Schreiberhände ${ }^{56}$ mit dem langjährigen, überwiegend nebenamtlich tätigen Notisten Johann Gottlieb Haußstädler. Als Gegenposition zu Fechner hat Landmann im Jahr 1999 eine Identität aller genannten Notisten propagiert, ${ }^{57}$ zehn Jahre später

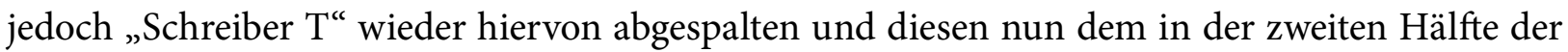
1760er Jahre neben Haußstädler als zweiten Notisten der Comédie françoise nachweisbaren Carl Samuel Zinnert zugeordnet, ${ }^{58}$ zugleich aber „AnonH1a/b“ und auch die wenigen Nachweise für „AnonH2a/b“ (dessen mutmaßliche Identität mit „Schreiber T“ zu diesem Zeitpunkt noch nicht erkannt war) als frühe Schriftstadien unter Haußstädler subsumiert. ${ }^{59}$ Es scheint daher angebracht, ausgehend von den Hasse-Belegen der Notisten „AnonH1“ und „AnonH2“ diese Problematik nochmals neu zu beleuchten.

52 Im Autograph der Karnevalsoper 1754 Artemisia in Form einiger Textunterlegungen (I-Mc: Part.Tr.ms. 164; vgl. auch Schmidt-Hensel, Hasse 2009 [wie Anm. 1], S. 141 Anm. 167); daneben ist auch die Partitur zu Eroe cinese D-Bsa: SA 1090, die wie oben dargestellt wahrscheinlich in relativer zeitlicher Nähe zur Erstaufführung im Oktober 1753 angefertigt wurde, von Balch geschrieben.

53 Vgl. Landmann, Drei Studien 22010 (wie Anm. 3), S. 141f., wonach Balch 1753/54 als „Musicus“ in der Hofhaltung des Grafen Brühl geführt wurde; Landmann verzeichnet als frühe Schreibarbeiten Balchs u.a. nachgefertigte Stimmen zu Lottis Credidi (in Mus.2159-E-8a), die sie auf „ca. 1751“ datiert (S. 142 und 334 ff., Abb. III.2-4).

54 Neben der oben in Anm. 52 genannten Eroe-cinese-Partitur sind dies: D-Leu: N.I. 10291 Artemisia (u.a. mit Kremmler und „AnonH1b“); D-Hami: 86, D-Bsa: SA 1116 und D-Dl: Mus.2477-F-81 Il re pastore (die beiden zuletzt genannten wiederum zusammen mit Kremmler); D-Dl: Mus.2477-F-7a Ezio-Orchesterstimmen (mit Grundig).

55 Vgl. hierzu Schmidt-Hensel, Hasse 2009 (wie Anm. 1), Bd. 1, S. 170 f.

56 Vgl. Fechner, Studien 1999 (wie Anm. 3), S. 133-135; leider fehlen hier jegliche Angaben zu Kriterien, anhand derer Fechner die auf den ersten Blick zweifellos sehr ähnlich wirkenden Schriftzüge voneinander unterschieden hat.

57 Landmann, Katalog 1999 (wie Anm. 10), S. 33, Anm. 51.

58 Landmann, Drei Studien 2010 (wie Anm. 3), S. 150 f. und 175 f.

59 Landmann, Drei Studien 2010 (wie Anm. 3), S. 150 f. 
In diesem Zusammenhang ist zunächst zu konstatieren, dass die Notisten „AnonH1“ und „AnonH2“ trotz gewisser Ähnlichkeiten in den Schriftbelegen der Jahre 1747/48 mit Sicherheit nicht unterschiedliche Schriftphasen ein und desselben Schreibers darstellen. Wie erwähnt, lassen sich bei näherer Betrachtung durchaus einige charakteristische Unterschiede feststellen; vor allem aber begegnen die Schriftzüge beider Notisten mehrfach in ein und derselben Quelle mit großer Stabilität in den jeweiligen Schreibanteilen, so dass diese Unterschiede weder auf ein zeitliches Nacheinander zurückzuführen sind noch mit der wechselnden Tagesform eines noch ungeübten Schreibers erklärt werden könnten. Spätestens 1750 / 51 treten dann die Unterschiede zwischen den beiden Schreiberhänden sehr deutlich zutage, indem „AnonH1(b)“ in seiner Schriftentwicklung andere Wege als „AnonH2“ alias „Schreiber T“ geht und sich auch insgesamt vom Mainstream der Dresdner Notisten der Jahrhundertmitte signifikant abhebt. Allerdings ist zu beachten, dass in einigen Einzelabschriften Hasse'scher Opernarien der 1750er Jahre ein weiterer Notist begegnet, der in manchen Details (z. B. der Form des Bass-Schlüssels mit waagrecht nach links gezogenem und am Ende nach oben gebogenem Balken) an „AnonH1b“ erinnert, in anderen Aspekten sich aber deutlich von diesem unterscheidet (z. B. Viertelpause mit Anstrich) ${ }^{60}$

Kann somit „AnonH1“ ausweislich der Schriftbelege ab 1750/51 weder mit Haußstädler noch mit einem der anderen Schreiber der Gruppe „Schreiber O / S / T“ identisch sein, so gestaltet sich die Frage einer Zuordnung von „AnonH2“ beziehungsweise „Schreiber T“ diffiziler. Problematisch ist in diesem Zusammenhang insbesondere, dass Haußstädlers Schrift im Laufe seiner langen Tätigkeit am Dresdner Hof höchstwahrscheinlich eine erhebliche Entwicklung durchlaufen hat, ohne dass die Chronologie der einzelnen Schriftstadien in jedem Fall als gesichert gelten kann. Sicher zu datieren sind Abschriften verschiedener Opern der 1760er bis 1780er Jahre, ${ }^{61}$ ferner etliche nachweislich in die 1770er fallende Kopiaturen von Klavierkonzerten ${ }^{62}$ und schließlich - sofern Fechners „Schreiber O“ mit Haußstädler identisch sein sollte - eine Reihe von Stimmensätzen zu Instrumentalmusik aus den Jahren 1751 bis $1758 .{ }^{63}$ Hingegen erscheinen die an verschiedener Stelle für den Haußstädler der späten 1740er und frühen 1750er Jahre gegebenen Schriftproben eher uneinheitlich und ermangeln nicht nur bisweilen einer plausiblen Begründung für die vorgeschlagene zeitliche Einordnung, sondern umfassen zum Teil auch deutlich divergierende Schriftbeispiele für mehr oder weniger dieselbe Zeit; ${ }^{64}$ gänzlich unbeachtet blieb im Übrigen bislang, dass Haußstädler offenbar zu einem noch nicht näher bekannten Zeitpunkt die Form seines c-Schlüssels änderte, der in den Belegen ab den 1760er Jahren drei senkrechte Striche, in den früheren hingegen lediglich deren zwei aufweist. ${ }^{65}$ Ein eindeutiges Urteil, ob die aus Hasse-Opern stammenden Belege von „AnonH2“ alias „Schreiber T“, die wie gezeigt sämtlich aus dem Zeitraum 1747/48 (bzw. im Falle der Demetrio-Partitur noch früher) bis 1750/ 51 stammen, frühe Schriftformen von

60 Beispielsweise D-Dl: Mus.2477-F-110,6 und -110,12.

61 Vgl. Landmann, Drei Studien 22010 (wie Anm. 3), Abb. III.47-50 (NB: Abb. III.44/45 zeigen - ebenso wie Abb. I.22 - den Notisten „AnonH1a“).

62 Vgl. Rosenmüller, Clavierkonzerte 2002 (wie Anm. 4), S. 63-70.

63 Vgl. Fechner, Studien 1999 (wie Anm. 3), S. 133 f.

64 Vgl. beispielsweise die beiden Partiturabschriften von Hasses Il natal di Giove Mus.2477-F-59 (vgl. Landmann, Katalog 1999 [wie Anm. 10], Abb. 22) und D-Dl: Mus.2477-F-58 (ebd., Abb. 23), die von Landmann auf „sehr früh (1749?)“ bzw. „1749/50“ geschätzt werden, aber im Duktus der Handschrift durchaus divergieren. 
Haußstädler sein könnten, erscheint angesichts dieser eher diffusen Vergleichsbasis für die frühe Zeit und des weiten zeitlichen Abstands zu den sicher datierbaren Schriftbelegen Haußstädlers nicht möglich. Ein Indiz, das eher gegen eine solche Identifizierung sprechen könnte, ist jedoch möglicherweise in der Form der Viertelpause zu sehen: Während in fast allen für Haußstädler gesicherten Schriftproben der Winkel zwischen Anstrich und Aufstrich deutlich größer als $90^{\circ}$ ist und der Aufstrich somit in aller Regel sozusagen ,weich“ aus dem Anstrich hervorgeht, zeigen die Viertelpausen von „AnonH2“ beziehungsweise „Schreiber T“ meist nur einen kurzen unteren Anstrich oder Ansatz, aus dem in einem mehr oder weniger rechten Winkel der meist ziemlich senkrechte, manchmal sogar leicht nach links geneigte Aufstrich entspringt; die gleiche Beobachtung scheint im Übrigen auch auf „Schreiber S“ zuzutreffen. Dass sich dieses Phänomen auch bei den von Landmann für Zinnert aus den 1760er Jahren mitgeteilten Schriftproben zeigt, könnte darauf hindeuten, dass wir es bei „AnonH2“ beziehungsweise „Schreiber T“ in der Tat mit frühen Schriftstadien dieses Notisten zu tun haben (der jedoch in den 1760 er Jahren offenbar ebenso wie der spätere Haußstädler einen abweichenden c-Schlüssel mit drei statt zwei senkrechten Strichen schrieb); ${ }^{66}$ auch hier erscheint aber die empirische Basis für eine solche Zuordnung über eine Lücke von rund 15 Jahren hinweg einstweilen nur bedingt tragfähig.

Ist somit nach derzeitiger Forschungslage keine sichere Identifizierung von „AnonH2“ möglich, so führt die Frage nach der Identität von „AnonH1“ vollends auf das Gebiet der Spekulation - einer Spekulation freilich, die sich geradezu aufdrängt: Auf der einen Seite ist nämlich mit „AnonH1“ ein Schreiber zu individualisieren, der zwischen 1745/46 und circa 1755 in Dresden eine erhebliche Schreibleistung erbracht hat und den Hasse offenbar als so zuverlässig schätzte, dass er ihm Eintragungen in seinen Autographen anvertraute. Auf der anderen Seite taucht wie erwähnt in den Akten mehrfach ein gewisser Joseph Leonhard Butz / Putz auf, der 1750 als Berufsbezeichnung „Notista“ angibt und für dessen mittelfristige Anstellung als Hofnotist sich Hasse 1754 nachdrücklich einsetzt, dem sich aber bislang keinerlei Kopialien haben zuordnen lassen. Insofern erscheint es zumindest denkbar, dass „AnonH1“ mit Butz/ Putz identisch sein könnte. Solange aber in den Akten nicht doch noch irgendwo ein eigenhändiges Schriftstück dieses Notisten auftaucht, wird sich diese Hypothese nicht verifizieren lassen, zumal es Indizien dafür zu geben scheint, dass sich hinter Butz / Putz auch einer der anderen bislang, namenlosen ' Dresdner Notisten der Jahrhundertmitte verbergen könnte. ${ }^{67}$

66 Vgl. Landmann, Drei Studien 22010 (wie Anm. 3), Abb. III.193-194 (nicht jedoch III.196).

67 So gibt es aus anderen Quellen auch Hinweise darauf, dass es sich bei „Schreiber S“ um Butz / Putz handeln könne; vgl. Katrin Bemmann, Wolfgang Eckhardt, Gerhard Poppe, Steffen Voss: „Über die Kopisten der im Schranck No: II aufbewahrten Manuskripte", in: Schranck No: II. Das erhaltene Instrumentalmusikrepertoire der Dresdner Hofkapelle aus den ersten beiden Dritteln des 18. Jahrhunderts, hrsg. von Gerhard Poppe unter Mitarbeit von Katrin Bemmann, Wolfgang Eckhardt, Sylvie Reinelt und Steffen Voss, Beeskow 2012, S. 191-229, hier S. 215 f. (Forum Mitteldeutsche Barockmusik. 2). 


\section{VII}

Wie gezeigt, lassen sich aus der Untersuchung der Dresdner Abschriften der Opern Johann Adolf Hasses eine Reihe von Erkenntnissen gewinnen, die sich zum Teil wie Mosaiksteinchen in das aus der instrumentalen Überlieferung gewonnene Bild einfügen, zum Teil dieses Bild erweitern und modifizieren helfen. Gerade im Zusammenhang mit den Anonymi „AnonH1“ und „AnonH2“/ „Schreiber T“ scheinen zugleich auch die methodischen Implikationen und Grenzen der Identifizierung und Zuordnung von Schreiberhänden auf. Insbesondere zeigt sich, dass in einem Umfeld von mehreren offenkundig recht ähnlich schreibenden Notisten, von denen zumindest einige über einen langen Zeitraum tätig waren und möglicherweise ihre Schriftformen im Laufe der Zeit deutlich verändert haben, eine eindeutige Individualisierung der einzelnen Schreiber nur schwer möglich erscheint. Insofern bleibt zu hoffen, dass sich durch die Einbeziehung der hier vorgestellten Schriftbelege in den Kontext der Überlieferung instrumentaler und geistlicher Werke weitere Hinweise auf die Identität dieser Notisten beziehungsweise auf eine Zusammengehörigkeit mit namentlich bereits identifizierten, bislang aber vor allem in späteren Schriftformen sicher belegten Notisten finden lassen. 